\title{
Energy Harvesting and Information Transmission Mode Design for Cooperative EH-Abled IoT Applications in beyond 5G Networks
}

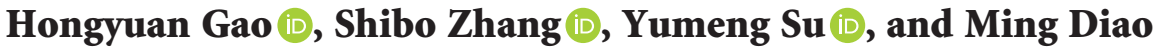 \\ College of Information and Communication Engineering, Harbin Engineering University, Harbin 150001, China \\ Correspondence should be addressed to Hongyuan Gao; gaohongyuan@hrbeu.edu.cn
}

Received 16 November 2019; Accepted 9 January 2020; Published 12 February 2020

Guest Editor: Juraj Gazda

Copyright ( $\odot 2020$ Hongyuan Gao et al. This is an open access article distributed under the Creative Commons Attribution License, which permits unrestricted use, distribution, and reproduction in any medium, provided the original work is properly cited.

\begin{abstract}
Energy harvesting (EH) technology is considered to be a promising approach to provide enough energy for energy-constrained Internet of Things (IoT). In this paper, we propose an energy harvesting and information transmission mode for the spectrum sharing system with cooperative EH-abled IoT applications in beyond 5G networks. Different from most existing IoT spectrumsharing research studies, in our system, both primary user (PU) and IoT devices (IDs) collect energy for their information transmission. In addition, for all IDs, they should realize two communication functions: working as relays to help the information transfer process of PU and completing their own information transmission. We analytically derive exact expressions for the throughput of the primary system and IoT system and then formulate two objective functions. It is easy to see that power splitting ratio, dynamic EH ratio, power sharing ratio, and relay selection should be optimized to get the best performance for different communication circumstances. Actually, it is a hybrid NP-hard problem to optimize these parameters and traditional algorithms cannot solve it well. Therefore, a novel algorithm-quantum whale optimization algorithm (QWOA) is proposed to obtain the best performance. Simulation results show the good performance of QWOA in different simulation scenarios.
\end{abstract}

\section{Introduction}

Internet of Things (IoT) is an emerging technique that can provide intelligent information exchange opportunity for smart devices in the future communication scenarios and the beyond $5 \mathrm{G}$ networks $[1,2]$. With the development of IoT, energy efficiency and spectrum efficiency have been two essential concerns due to the rapidly growing number of IoT devices $[3,4]$. Since the technique of IoT spectrum sharing is able to make better use of the spectrum resource, many experts and scholars have a deep study on it then apply to IoT systems [5-8]. Some IoT spectrum-sharing protocols are proposed in [9-11]. In the investigated IoT system, the licensed primary user (PU) can use its own spectrum if it needs at any time, and IoT devices (IDs) can share the PU's spectrum in the case that IDs have no worse effect on the PU. In [10], Khan et al. studied the spectrum-sharing IoT structure. Some future application scenarios, such as smart grid, smart city, and wireless sensor are investigated. An arbitrarily-shaped underlay cognitive network was investigated by Guo et al. [11]. To satisfy interference constraint and meet outage probability of the PU, a cooperative protocol was proposed. In [12], Aslam et al. designed opportunistic spectrum sharing protocol, frame structure, and energy consumption function.

In order to prolong the life of the network, $\mathrm{EH}$ is seen as an effective technique which is widely applied in energylimited environment, where the batteries of communication devices are hard to recharge or replace $[13,14]$. EH technique can supply enough energy for cooperative communication, information transfer, and many other telecommunication works [15-17]. To explore potential of EH, many study works have been conducted in recent years $[18,19]$. To satisfy energy demand for the communication system, a fixed harvesting energy ratio was considered, and the communication system could harvest energy in one time slot for information transfer 
in [20-22]. In [21], Gao et al. investigated an energy harvesting cooperative communication protocol. Network users can collect energy from ambient environment and complete their own information transmission. In [22], relay nodes can harvest energy from wireless signals. Maji et al. presented the mathematical expressions for the system outage probability.

The combination of IoT and $\mathrm{EH}$, which is aimed to exert advantage of them, has been a hot topic in recent years $[23,24]$. It is necessary to investigate the EH-IoT applications because some IoT scenarios are limited by energy constraint and cannot have a good performance [25-27]. An EH-abled IoT system which consists of IoT devices and an access point were considered by Lee and Lee [26]. Hybrid energy resources, i.e., wireless signals and on-grid energy resource were combined to realize information transmission. Gurjar et al. [27] proposed an energy transfer approach in an overlay cognitive IoT system. The system throughput, outage probability, and energy efficiency were investigated. Human kinetic energy is also seen as an energy resource by Ju et al. [28]. The power allocation structure, sink selection, and power control blocks were discussed. Research studies [26-28] illustrated the effectiveness of $\mathrm{EH}$ technology in IoT applications.

For improving quality of service (QoS) and extend network communication range, relay cooperative communication is an essential technique in modern communication which can be combined with spectrum-sharing networks to further improve the performance [29-32]. Some works technically studied the relay selection problem for cooperative networks [33-35]. In [33], to measure performance of the system more comprehensively, Atapattu et al. considered the minimum achievable rate of the system. To further exploit benefits of relay cooperative communication, there are more and more studies on relay selection problem in recent years. For maximizing sum rate for the whole network in a multiuser network, Sharma et al. [35] presented a relay selection protocol for the cooperative ad hoc network. The optimization problem was assigning available relay nodes for different communication nodes. However, common channel interference (CCI) was not considered in $[33,35]$. To find a relay selection scheme with CCI, Singh et al. [34] presented a new relay selection scheme considering CCI among multiple users. However, the performance of the proposed protocol is not optimal solution and only a suboptimal solution is obtained.

Most existing works as mentioned above mainly discussed about designing a better system of spectrumsharing IoT $[8,10,12]$ or EH-abled IoT $[19,27,28]$. The research studies about cooperative information exchange in EH-abled IoT with spectrum sharing are few. However, for some IoT distance transmission situations, cooperative relay transmission is necessary and can greatly improve network performance $[29,36,37]$. Developing the cooperative information transmission mode for the EHabled IoT with spectrum sharing is essential. Besides, considering the problem of existing wireless $\mathrm{EH}$ instability (susceptible to the channel gain), it is necessary to design a dynamic EH time slot structure to collect energy (ensuring energy supply). A dynamic EH time slot structure can maintain the stability of system information transmission and $\mathrm{EH}$.

In this paper, we consider a cooperative EH-abled IoT with spectrum sharing (CEH-IoT-SS), where each ID can not only share the licensed spectrum of the PU to transmit its own information but also can help PU's information transmission. For different practical requirements, two objective functions are formulated and the main contribution of this paper can be summarized as follows:

(i) We propose a novel CEH-IoT-SS model for existing IoT applications. Different from models in existence, there are multiple IDs and single PU in CEH-IoT-SS and some IDs can work as relays to help PU's information transfer. At the same time, each ID transmits its own information during one time slot. The PU is composed of a primary transmitter (PT) and a primary receiver (PR). The PT collects energy from ambient energy source while each IoT device transmitter (IDT) harvests energy from ambient energy source and a portion of received PT's signal. The energy harvested from PT's signal for each ID can be seen as a transmission energy supplement.

(ii) We derive analytical expressions for the throughput of primary network and IDs. Two objective functions are given for different communication situations. Analytical expression indicates that the scheme of power splitting ratio, dynamic $\mathrm{EH}$ ratio, power sharing ratio, and relay selection should be optimized.

(iii) A new algorithm-quantum whale optimization algorithm (QWOA) is designed to optimize problems which are proposed. Actually, finding the relay selection scheme is a discrete optimization problem. However, the process for obtaining the best power splitting ratio, power sharing ratio, and dynamic $\mathrm{EH}$ ratio is solving a continuous optimization problem. Since it is a hybrid optimization problem, traditional continuous or discrete algorithms cannot get the best solution. Therefore, QWOA is proposed to solve it. Through simulation results, we can see QWOA has advantages which traditional algorithms do not have.

Other parts for this paper are structured in the following way. In Section 2, we will give our proposed CEH-IoT-SS model and time slot structure. For Section 3, expressions of throughput for primary network and IDs are derived. For different communication scenarios, two objective functions are formulated. In Section 4, QWOA is proposed to find the best power splitting ratio, dynamic $\mathrm{EH}$ ratio, power sharing ratio, and relay selection scheme for different communication scenarios. Simulation results and conclusion will be provided in Sections 5 and 6. 


\section{System Model and Time Slot Structure}

In this part, a CEH-IoT-SS is considered. As shown in Figure 1, the primary network consists of one PT and one PR. PT tries to deliver information to PR. We consider the situation where PT cannot transmit information to PR directly so PT needs some relays to complete information transfer [27]. $M$ pairs of IDs, which consist of $M$ IDTs and $M$ IoT device receivers (IDRs), implement two communication functions: (1) some IDTs serve as relays to help PT transmit information; (2) each IDT has its own information for transmitting to its corresponding IDR. Both PT and IDTs can harvest energy from ambient energy sources to complete their own information transmission. Besides, IDTs can scavenge energy from a portion of received signal from PT as transmission energy supplement. In the proposed CEH-IoTSS, energy storage equipment can be a super capacitor and there is no energy rested at the end of each time slot $[21,38]$.

In this model, each information channel follows Rayleigh distribution, and parameters of different Rayleigh distributions are related with distance between two devices. The channel state information (CSI) between two devices is the same during the same time slot. Meanwhile, two channels are mutual independent from each other in one time slot and CSI of the same channel in two different time slots is independent. CSI from PT to the $m$ th IDT $(m=1,2, \ldots, M)$ is represented as $G_{\mathrm{PT}_{\text {,IDT }}}$, CSI from PT to the $m$ th IDR is represented as $G_{\mathrm{PT}, I D R_{m}}$, CSI from the $m$ th IDT to the $m$ th IDR is represented as $G_{\mathrm{IDT}_{m}, \mathrm{IDR}_{m}}$, and CSI from the $m$ th IDT to PR is represented as $G_{\mathrm{IDT}_{m}, \mathrm{PR}}$.

To simplify representation of the distance between two communication devices, we make definition as follows: the distance between PT and the $m$ th IDT is denoted as $d_{\mathrm{PT}, \mathrm{IDT}}$. Similarly, the distance between the PT and the $m$ th IDR is denoted as $d_{\mathrm{PT}_{\text {TIDR }},}$, the distance between the $m$ th IDT to the $m$ th IDR is denoted as $d_{\mathrm{IDT}_{m}, \mathrm{IDR}_{m}}$, and the distance between the $m$ th IDT and PR is denoted as $d_{\mathrm{IDT}_{m}, \mathrm{PR}}$. The CSI $G_{\mathrm{PT}, \mathrm{IDT}}{ }_{m}$, $G_{\mathrm{PT}, I D R_{m}}, G_{\mathrm{IDT}_{m}, \mathrm{IDR}_{m}}$, and $G_{\mathrm{IDT}_{m}, \mathrm{PR}}$ follow exponentially distribution with parameters $d_{\mathrm{PT}, \mathrm{IDT}}^{-\ell}, d_{\mathrm{PT}^{2} \mathrm{IDR}_{m}}^{-\ell}, d_{\mathrm{IDT}_{m}, \mathrm{IDR}_{m}}^{-\ell}$, and $d_{\mathrm{IDT}}^{-\ell}, \mathrm{PR}$, respectively. $\ell$ is the factor of path loss. For any two different exponentially distribution, it is independent from each other. In proposed CEH-IoT-SS, each PT transmits its information to PR by cooperative communication with IDTs, and IDTs also transmit their own information to corresponding IDRs. In addition, both PT and IDTs collect energy for information transmission. Figure 2 shows one time slot of EH and information transmission mode for the proposed CEH-IoT-SS. Each time slot for the CEH-IoT-SS is composed of three phases: (1) in the first phase $(0, \alpha T]$, both PT and IDTs harvest energy from ambient energy source, $\alpha$ is dynamic EH ratio, $0<\alpha<1$, and $T$ is the duration of one time slot. (2) In the second phase $(\alpha T,(1+\alpha) T / 2]$, PT transmits its information to IDTs and IDRs. One portion of each IDT's received signal is used for cooperative communication. The other portion of received signal is the collected energy by each IDT as the transmission energy supplement. (3) For the third phase $((1+\alpha) T / 2, T]$, each IDT which is selected as a relay will transmit its own signal and received signal to IDR and PR simultaneously. Other IDTs transmit their own signals to their corresponding IDRs.

\section{Throughput Analysis and Objective Function Design}

This part we will derive expressions for the throughput of primary network and IDs in CEH-IoT-SS. For different communication needs, we formulate two objective functions.

3.1. EH of PT and IDT in the First Phase. For the first phase, both PT and IDTs collect energy from ambient energy source. The length of the first phase is $\alpha T$, so energy stored by $\mathrm{PT}$ and IDTs can be shown as

$$
E_{\mathrm{PT}}=Y_{\mathrm{PT}} \alpha T,
$$

where $Y_{\mathrm{PT}}$ is $\mathrm{EH}$ rate of $\mathrm{PT}[38,39]$.

Similarly, the energy stored for the $m$ th IDT can be calculated as

$$
E_{\mathrm{IDT}_{m}}^{T_{1}}=\alpha T Y_{\mathrm{IDT}_{m}}
$$

where $Y_{\mathrm{IDT}_{m}}$ is $\mathrm{EH}$ rate of the $m$ th IDT. The $\mathrm{EH}$ process of PT is ended and PT no longer collects energy in one time slot. However, IDTs will still gather energy in the second phase. Since all the harvested energy is used up in one time slot, so transmit power provided by PT can be shown as

$$
P_{\mathrm{PT}}=\frac{E_{\mathrm{PT}}}{(1-\alpha) T / 2}=\frac{2 Y_{\mathrm{PT}} \alpha}{1-\alpha} \text {. }
$$

3.2. Information Transmission of PT in the Second Phase. In the second phase for one time slot, PT transmits its signal $x$ to IDTs and IDRs, where $x$ is normalized as $E|x|^{2}=1$. The received signals at the $m$ th $(m=1,2, \ldots, M)$ IDT and the $m$ th IDR are shown as follows:

$$
\begin{aligned}
& y_{\mathrm{IDT}_{m}}=\sqrt{P_{\mathrm{PT}}} G_{\mathrm{PT}, \mathrm{IDT}_{m}} x+n_{1}, \\
& y_{\mathrm{IDR}_{m}}=\sqrt{P_{\mathrm{PT}}} G_{\mathrm{PT}, \mathrm{IDR}_{m}} x+n_{2},
\end{aligned}
$$

where $n_{1}$ is additive white Gaussian noise (AWGN), in which power is $w_{1}$, and $n_{2}$ is AWGN with power $w_{2}$.

On the basis of power splitting approach, the power splitting circuit of the $m$ th IDT will divide the received signal into two parts, $\sqrt{\beta} y_{\mathrm{IDT}_{m}}$ and $\sqrt{1-\beta} y_{\mathrm{IDT}_{m}}$, which is used for $\mathrm{EH}$ and information transfer, respectively. $\beta(0<\beta<1)$ is the power splitting ratio. Hence, the portion of the received signal for $\mathrm{EH}$ can be given by

$$
y_{\mathrm{IDT}_{m}}^{\mathrm{EH}}=\sqrt{\beta} y_{\mathrm{IDT}_{m}}=\sqrt{P_{\mathrm{PT}} \beta} G_{\mathrm{PT}_{, \mathrm{IDT}} m} x+n_{1} \sqrt{\beta} .
$$




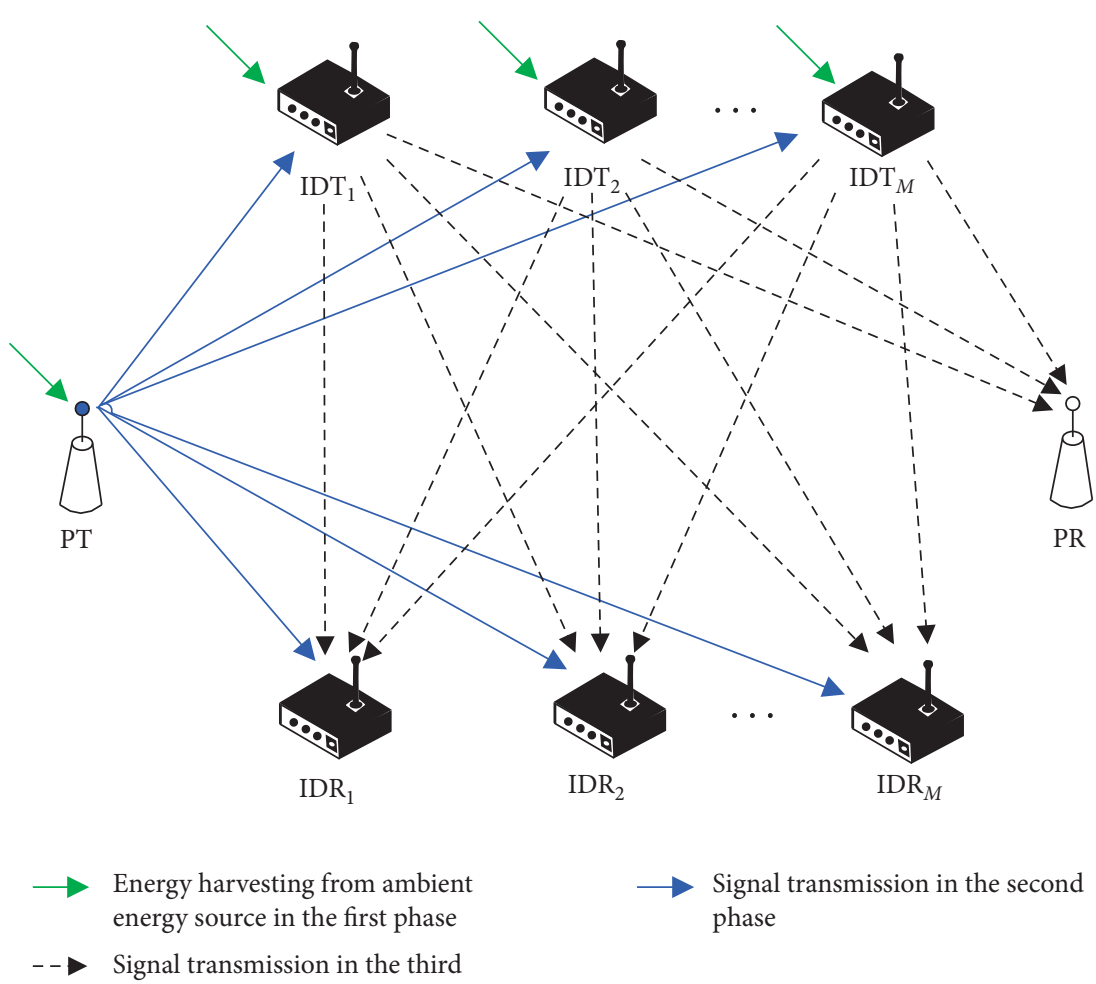
phase

FIGURE 1: System model of CEH-IoT-SS.

PT
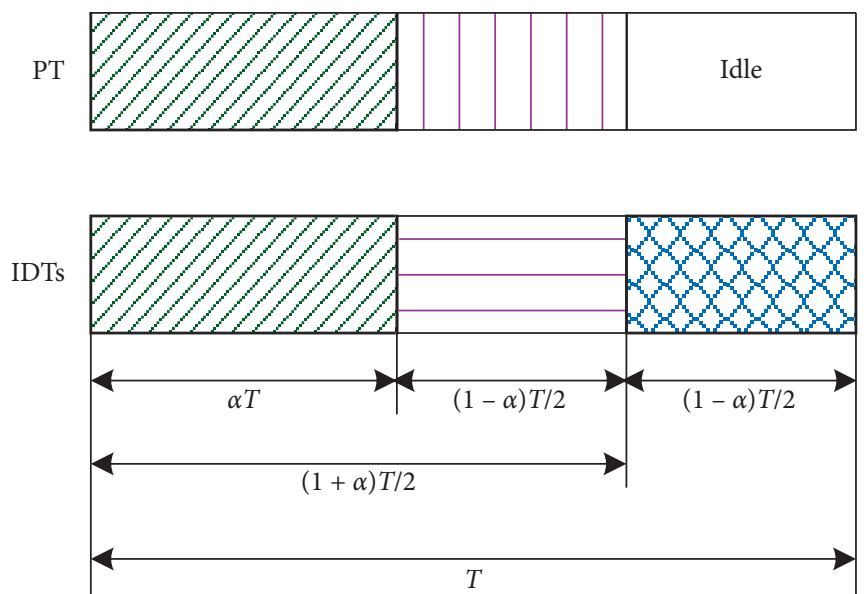

PT and IDTs harvest and store energy from the ambient environment

IDTs receive the signal from $\mathrm{PT}$ and collect energy from a portion of the received signal.The other portion of the received signal is use for cooperative communication

1] PT transmit signals to IDTs and IDRs

IDTs which are selected as relays transmit the received signals of PT to PR and other IDTs transfer their own signal to IDRs

Figure 2: Structure of time slot. 
Energy stored by the $m$ th IDT at the second phase is calculated as

$$
E_{\mathrm{IDT}_{m}}^{T_{2}}=\frac{(1-\alpha) T}{2} \rho P_{\mathrm{PT}} \beta\left|G_{\mathrm{PT}, \mathrm{IDT}_{m}}\right|^{2}=T \rho Y_{\mathrm{PT}} \alpha \beta\left|G_{\mathrm{PT}, \mathrm{IDT}_{m}}\right|^{2},
$$

where $\rho$ is the efficiency of EH. Since collected energy from AWGN is small, it can be neglected. Therefore, total energy stored by the $m$ th IDT in the first two phases can be shown as

$$
E_{\mathrm{IDT}_{m}}=E_{\mathrm{IDT}_{m}}^{T_{1}}+E_{\mathrm{IDT}_{m}}^{T_{2}}=\alpha T Y_{\mathrm{IDT}_{m}}+T \rho Y_{\mathrm{PT}} \alpha \beta\left|G_{\mathrm{PT}, \mathrm{IDT}_{m}}\right|^{2} .
$$

The $m$ th IDT utilizes all the harvested energy in the third phase to complete cooperative communication and its own information transfer, so the power at the $m$ th IDT can be given by

$$
P_{\mathrm{IDT}_{m}}=\frac{E_{\mathrm{IDT}_{m}}}{(1-\alpha) T / 2}=\frac{2 \alpha Y_{\mathrm{IDT}_{m}}+2 \rho Y_{\mathrm{PT}} \alpha \beta\left|G_{\mathrm{PT}, \mathrm{IDT}_{m}}\right|^{2}}{1-\alpha} .
$$

On the other hand, the portion of the received signal at the $m$ th IDT for information transfer is as follows:

$$
\begin{aligned}
y_{\mathrm{IDT}_{m}}^{\mathrm{IT}}= & \sqrt{1-\beta} y_{\mathrm{IDT}_{m}}+n_{3}=\sqrt{P_{\mathrm{PT}}(1-\beta)} G_{\mathrm{PT}, \mathrm{IDT}_{m}} x \\
& +n_{1} \sqrt{(1-\beta)}+n_{3},
\end{aligned}
$$

where $n_{3}$ is AWGN which is caused by the signal conversion from the passband to baseband [40] at the $m$ th IDT and the power of $n_{3}$ is $w_{3}$.

The received signal at the $m$ th IDT is needed to be normalized, and the power normalization factor $\psi_{m}$ is in the following:

$$
\psi_{m}=\frac{1}{\sqrt{P_{\mathrm{PT}}(1-\beta)\left|G_{\mathrm{PT}, \mathrm{IDT}}\right|^{2}+(1-\beta) w_{1}+w_{3}}} .
$$

The received normalization signal of the $m$ th IDT is

$$
\begin{aligned}
& y_{\mathrm{IDT}_{m}}^{\mathrm{IT}-\mathrm{Norm}}=\psi_{m} y_{\mathrm{IDT}_{m}}^{\mathrm{IT}}=\frac{\sqrt{\left(2 Y_{\mathrm{PT}} \alpha /(1-\alpha)\right)(1-\beta)} G_{\mathrm{PT}, \mathrm{IDT}_{m}}}{\sqrt{\left(2 Y_{\mathrm{PT}} \alpha /(1-\alpha)\right)(1-\beta)\left|G_{\mathrm{PT}, \mathrm{IDT}_{m}}\right|^{2}+(1-\beta) w_{1}+w_{3}}} x \\
& +\frac{\sqrt{(1-\beta)}}{\sqrt{\left(2 Y_{\mathrm{PT}} \alpha /(1-\alpha)\right)(1-\beta)\left|G_{\mathrm{PT}, \mathrm{IDT}_{m}}\right|^{2}+(1-\beta) w_{1}+w_{3}}} n_{1} \\
& +\frac{1}{\sqrt{\left(2 Y_{\mathrm{PT}} \alpha /(1-\alpha)\right)(1-\beta)\left|G_{\mathrm{PT}, \mathrm{IDT}_{m}}\right|^{2}+(1-\beta) w_{1}+w_{3}}} n_{3} .
\end{aligned}
$$

3.3. Information Transmission of IDT in the Third Phase. In the third phase, some IDTs will be selected as relays. If the $m$ th IDT is selected as a relay, the $m$ th IDT transmits the received normalized-signal $y_{\mathrm{IDT}}^{\mathrm{IT}-\mathrm{Norm}}$ and its own signal $z_{\mathrm{IDT}_{m}}$ by superposition coding in the following:

$$
z_{m}=\sqrt{\sigma} y_{\mathrm{IDT}_{m}}^{\mathrm{IT}^{\mathrm{T}} \mathrm{Norm}}+\sqrt{1-\sigma} z_{\mathrm{IDT}_{m}},
$$

where $\sigma(0<\sigma<1)$ is power sharing ratio, $z_{m}$ is the combined signal which is divided into two parts. The first part $\sqrt{\sigma} y_{\mathrm{IDT}_{m}}^{\mathrm{IT}-\mathrm{Norm}}$ is used to transmit PT's signal, and the second part $\sqrt{1-\sigma} z_{\mathrm{IDT}_{m}}$ is used to transmit IDT's own information. If the $m$ th IDT is not selected as a relay, it just needs to transmit its own information to its corresponding IDR:

$$
z_{m}=z_{\mathrm{IDT}_{m}}
$$

According to different communication functions of IDT, the signal transferred by the $m$ th IDT can be shown as 


$$
z_{m}= \begin{cases}\sqrt{\sigma} y_{\mathrm{IDT}_{m}}^{\mathrm{IT}-\mathrm{Norm}}+\sqrt{1-\sigma} z_{\mathrm{IDT}_{m}}, & \text { if } \mathrm{IDT}_{m} \text { is selected as relay, } \\ z_{\mathrm{IDT}_{m}}, & \text { if } \mathrm{IDT}_{m} \text { is not selected as relay. }\end{cases}
$$

Therefore, the received signal at $\mathrm{PR}$ is given by

$$
\begin{aligned}
& z_{\mathrm{PR}}=\sum_{m=1}^{M} \sqrt{P_{\mathrm{IDT}_{m}}} G_{\mathrm{IDT}_{m}, \mathrm{PR}} z_{m}+n_{4} \\
& =\sum_{m=1}^{M} b_{m} \frac{\sqrt{\left(2 Y_{\mathrm{PT}} \alpha /(1-\alpha)\right) \sigma P_{\mathrm{IDT}_{m}}(1-\beta)} G_{\mathrm{IDT}_{m}, \mathrm{PR} G_{\mathrm{PT}_{\mathrm{IDT}}}}}{\sqrt{\left(2 Y_{\mathrm{PT}} \alpha /(1-\alpha)\right)(1-\beta)\left|G_{\mathrm{PT}_{\mathrm{IDT}} \mathrm{IDT}_{m}}\right|^{2}+(1-\beta) w_{1}+w_{3}}} x \\
& +\sum_{m=1}^{M} b_{m} \frac{\sqrt{\sigma P_{\mathrm{IDT}_{m}}(1-\beta)} G_{\mathrm{IDT}_{m}, \mathrm{PR}}}{\sqrt{\left(2 Y_{\mathrm{PT}} \alpha /(1-\alpha)\right)(1-\beta)\left|G_{\mathrm{PT}_{\mathrm{IDT}}}\right|^{2}+(1-\beta) w_{1}+w_{3}}} n_{1} \\
& +\sum_{m=1}^{M} b_{m} \frac{\sqrt{\sigma P_{\mathrm{IDT}_{m}}} G_{\mathrm{IDT}_{m}, \mathrm{PR}}}{\sqrt{\left(2 Y_{\mathrm{PT}} \alpha /(1-\alpha)\right)(1-\beta)\left|G_{\mathrm{PT}_{\mathrm{IDT}}}\right|^{2}+(1-\beta) w_{1}+w_{3}}} n_{3} \\
& +\sum_{m=1}^{M} b_{m} \sqrt{P_{\mathrm{IDT}_{m}}(1-\sigma)} G_{\mathrm{IDT}_{m}, \mathrm{PR}_{\mathrm{IDT}_{m}}} \\
& +\sum_{m=1}^{M}\left(1-b_{m}\right) G_{\mathrm{IDT}_{m}, \mathrm{PR}} \sqrt{P_{\mathrm{IDT}_{m}}} z_{\mathrm{IDT}_{m}}+n_{4},
\end{aligned}
$$

where $b_{m}(m=1,2, \ldots, M)$ shows whether the $m$ th IDT is selected as a relay for information transfer of PT. If the $m$ th IDT is selected as a relay, $b_{m}=1$. The relay selection scheme of IDTs can be shown as $\mathbf{b}=\left[b_{1}, b_{2}, \ldots, b_{M}\right]$. If the $m$ th IDT is not chosen, $b_{m}=0 . n_{4}$ is AWGN with power $w_{4} \cdot \sum_{m=1}^{M} b_{m}\left[\sqrt{\sigma P_{\mathrm{IDT}_{m}}(1-\beta)} G_{\mathrm{IDT}_{m}, \mathrm{PR}} /\right.$ $\left.\sqrt{\left(2 Y_{\mathrm{PT}} \alpha /(1-\alpha)\right)(1-\beta)\left|G_{\mathrm{PT}_{\mathrm{IDT}}}\right|^{2}+(1-\beta) w_{1}+w_{3}}\right] n_{1}, \quad \sum_{m=1}^{M} b_{m}$

According to (9) and (17), the throughput of PR, i.e., the throughput of the primary network can be shown as follows: 


$$
\begin{aligned}
& R_{\mathrm{PR}}=\frac{1-\alpha}{2} \log _{2}\left(1+\gamma_{\mathrm{PR}}\right)=\frac{1-\alpha}{2} \log _{2}\left(1+\frac{\text { Signal }_{\mathrm{PR}}}{\text { Interference }_{\mathrm{PR}}}\right), \\
& \operatorname{Signal}_{\mathrm{PR}}=\left(\sum_{m=1}^{M} b_{m} \frac{\sqrt{\left(\left(4 \alpha Y_{\mathrm{IDT}_{m}}+4 \rho Y_{\mathrm{PT}} \alpha \beta\left|G_{\mathrm{PT}_{\mathrm{IDT}}}\right|^{2}\right) /(1-\alpha)^{2}\right) Y_{\mathrm{PT}} \alpha \sigma(1-\beta)} G_{\mathrm{IDT}_{m}, \mathrm{PR} G_{\mathrm{PT}_{\mathrm{IDT}}}}}{\sqrt{\left(2 Y_{\mathrm{PT}} \alpha /(1-\alpha)\right)(1-\beta)\left|G_{\mathrm{PT}_{\mathrm{IDT}}}\right|^{2}+(1-\beta) w_{1}+w_{3}}}\right)^{2}, \\
& \text { Interference }_{\mathrm{PR}}=\sum_{m=1}^{M} b_{m} \frac{\left(\left(2 \alpha Y_{\mathrm{IDT}_{m}}+2 \rho Y_{\mathrm{PT}} \alpha \beta\left|G_{\mathrm{PT}, \mathrm{IDT}_{m}}\right|^{2}\right) /(1-\alpha)\right) w_{1} \sigma(1-\beta)\left|G_{\mathrm{IDT}_{m}, \mathrm{PR}}\right|^{2}}{\left(2 Y_{\mathrm{PT}} \alpha /(1-\alpha)\right)(1-\beta)\left|G_{\mathrm{PT}, \mathrm{IDT}_{m}}\right|^{2}+(1-\beta) w_{1}+w_{3}} \\
& +\sum_{m=1}^{M} b_{m} \frac{\left(\left(2 \alpha Y_{\mathrm{IDT}_{m}}+2 \rho Y_{\mathrm{PT}} \alpha \beta\left|G_{\mathrm{PT}, \mathrm{IDT}_{m}}\right|^{2}\right) /(1-\alpha)\right) w_{3} \sigma\left|G_{\mathrm{IDT}_{m}, \mathrm{PR}}\right|^{2}}{\left(2 Y_{\mathrm{PT}} \alpha /(1-\alpha)\right)(1-\beta)\left|G_{\mathrm{PT}_{\mathrm{IDT}}}\right|^{2}+(1-\beta) w_{1}+w_{3}} \\
& +\sum_{m=1}^{M} b_{m} \frac{2 \alpha Y_{\mathrm{IDT}_{m}}+2 \rho Y_{\mathrm{PT}} \alpha \beta\left|G_{\mathrm{PT}, \mathrm{IDT}_{m}}\right|^{2}}{1-\alpha}(1-\sigma)\left|G_{\mathrm{IDT}_{m}, \mathrm{PR}}\right|^{2} \\
& +\sum_{m=1}^{M}\left(1-b_{m}\right)\left|G_{\mathrm{IDT}_{m}, \mathrm{PR}}\right|^{2} \frac{2 \alpha Y_{\mathrm{IDT}_{m}}+2 \rho Y_{\mathrm{PT}} \alpha \beta\left|G_{\mathrm{PT}, \mathrm{IDT}_{m}}\right|^{2}}{1-\alpha}+w_{4} .
\end{aligned}
$$

Similarly, the average throughput of $M$ IDT-IDR transmission pairs in CEH-IoT-SS is expressed as follows:

$$
\begin{aligned}
& R_{\mathrm{IDT}_{\mathrm{IDR}} \text { average }}=\frac{1-\alpha}{2 M} \sum_{m=1}^{M} \log _{2}\left(1+\frac{\mathrm{Signal}_{\mathrm{IDR}_{m}}}{\text { Interference }_{\mathrm{IDR}}}\right), \\
& \text { Signal }_{\mathrm{IDR}_{m}}=\left\{\begin{array}{cl}
\frac{2 \alpha Y_{\mathrm{IDT}_{m}}+2 \rho Y_{\mathrm{PT}} \alpha \beta\left|G_{\mathrm{PT}_{\mathrm{IDT}}}\right|^{2}}{1-\alpha}\left|G_{\mathrm{IDT}_{m}, \mathrm{IDR}_{m}}\right|^{2}, & \text { if } \mathrm{IDT}_{m} \text { is not selected as relay, } \\
\frac{2 \alpha Y_{\mathrm{IDT}_{m}}+2 \rho Y_{\mathrm{PT}} \alpha \beta\left|G_{\mathrm{PT}_{\mathrm{IDT}}}\right|^{2}}{1-\alpha}(1-\sigma)\left|G_{\mathrm{IDT}_{m}, \mathrm{IDR}_{m}}\right|^{2}, & \text { if } \mathrm{IDT}_{m} \text { is selected as relay, }
\end{array}\right. \\
& \text { Interference }_{\mathrm{IDR}_{m}}=\sum_{i=1, i \neq m}^{M} b_{i}\left|G_{\mathrm{IDT}_{i}, \mathrm{IDR}_{m}}\right|^{2 \alpha \alpha Y_{\mathrm{IDT}_{i}}+2 \rho Y_{\mathrm{PT}} \alpha \beta\left|G_{\mathrm{PT}, \mathrm{IDT}_{i}}\right|^{2}}(1-\sigma) \\
& +\sum_{i=1, i \neq m}^{M} b_{i} \frac{\sigma\left(\left(2 \alpha Y_{\mathrm{IDT}_{i}}+2 \rho Y_{\mathrm{PT}} \alpha \beta\left|G_{\mathrm{PT}, \mathrm{IDT}_{i}}\right|^{2}\right) / 1-\alpha\right)(1-\beta)\left|G_{\mathrm{IDT}_{i}, \mathrm{IDR}_{m}}\right|^{2} w_{1}}{\left(2 Y_{\mathrm{PT}} \alpha /(1-\alpha)\right)(1-\beta)\left|G_{\mathrm{PT}, \mathrm{IDT}}\right|_{i}+(1-\beta) w_{1}+w_{3}} \\
& +\sum_{i=1, i \neq m}^{M} b_{i} \frac{\sigma\left(\left(2 \alpha Y_{\mathrm{IDT}_{i}}+2 \rho Y_{\mathrm{PT}} \alpha \beta\left|G_{\mathrm{PT}_{1} \mathrm{IDT}_{i}}\right|^{2}\right) /(1-\alpha)\right)\left|G_{\mathrm{IDT}_{i}, \mathrm{IDR}_{m}}\right|^{2} w_{3}}{\left(2 Y_{\mathrm{PT}} \alpha / 1-\alpha\right)(1-\beta)\left|G_{\mathrm{IDT}_{i}, \mathrm{IDR}_{m}}\right|^{2}+(1-\beta) w_{1}+w_{3}} \\
& +\sum_{i=1, i \neq m}^{M}\left(1-b_{i}\right)\left|G_{\mathrm{IDT}_{i}, \mathrm{IDR}_{m}}\right|^{2 \alpha Y_{\mathrm{IDT}_{i}}+2 \rho Y_{\mathrm{PT}} \alpha \beta\left|G_{\mathrm{PT}, \mathrm{IDT}_{i}}\right|^{2}}+w_{5} \text {, }
\end{aligned}
$$


where $w_{5}$ is the AWGN power.

Proof. See Appendix.

3.4. Objective Function Design for Different Communication Requirements. According to the throughput results of the primary network and IDT-IDR transmission pairs, we design two objective functions for improving the data transmission rate in different communication scenarios.

\subsubsection{Objective Function of IDT-IDR Transmission Pairs'} Average Throughput with Primary Network Constraint. For this communication scenario, we aim to maximize the average throughput of IDT-IDR transmission pairs. At the same time, the performance of the primary network should satisfy requirement. That is to say, throughput of the primary network should be higher than a certain value. According to (18), we can find that the IDT-IDR transmission pairs' throughput is impacted by power splitting ratio, dynamic $\mathrm{EH}$ ratio, power sharing ratio, and relay selection scheme. Therefore, we focus on optimizing these parameters to maximize the primary network. Under this constraint of the primary network, we aim to maximize the average throughput of IDT-IDR transmission pairs. Combine (18) and (19), objective function can be given by

$$
\begin{aligned}
& \max \quad R_{\mathrm{IDT} \mathrm{IDR}_{\text {average }}}(\mathbf{b}, \alpha, \beta, \sigma) \\
& \text { subject to (1) } b_{m} \in\{0,1\} \\
& \text { (2) } 0<\alpha<1 \\
& \text { (3) } 0<\beta<1 \\
& \text { (4) } 0<\sigma<1 \\
& \text { (5) } R_{\mathrm{PR}}^{\text {require }} \leq R_{\mathrm{PR}} \text {, }
\end{aligned}
$$

where $R_{\mathrm{PR}}^{\text {require }}$ is threshold that the throughput of the primary network should achieve.

3.4.2. Objective Function of Sum Throughput with Primary Network Constraint. In this situation, we aim to maximize sum throughput of the whole network with constraint of the primary network. Optimal power splitting ratio, dynamic $\mathrm{EH}$ ratio, power sharing ratio, and relay selection scheme should be found and objective function of sum throughput is formulated as follows:

$$
\begin{array}{ll}
\max & R_{\text {sum }}(\mathbf{b}, \alpha, \beta, \sigma)=R_{\mathrm{PR}}+R_{\mathrm{IDT}-\mathrm{IDR}_{\text {average }}} \\
\text { subject to } & \text { (1) } b_{m} \in\{0,1\} \\
& (2) 0<\alpha<1 \\
& \text { (3) } 0<\beta<1 \\
& \text { (4) } 0<\sigma<1 \\
& \text { (5) } R_{\mathrm{PR}}^{\text {require }} \leq R_{\mathrm{PR}} .
\end{array}
$$

To obtain the best throughput of two communication circumstances proposed above is the process for solving the hybrid NP-hard optimization problem [41]. However, traditional intelligent algorithms cannot solve it well because of slow convergence speed and poor convergence accuracy. Hence, we proposed a novel algorithm-QWOA.

\section{Optimization for Throughput Based on Quantum Whale Optimization Algorithm}

For the problems which are proposed above, relay selection is a discrete optimization problem. However, getting the best power splitting ratio, dynamic $\mathrm{EH}$ ratio, and power sharing ratio is a progress of finding continuous solution. Since domains of them are different and dimensions are complex, traditional intelligent algorithms cannot be easily applied to get the optimal solution. Hence, a new intelligent algorithmquantum whale optimization algorithm (QWOA) is proposed to solve it.

4.1. QWOA for Hybrid Optimization Problem. QWOA combines the advantages of whale optimization algorithm (WOA) [42] and quantum evolution theory of quantum computing [43-45]. Since relay selection is a discrete optimization problem and obtaining other parameters is a continuous optimization problem, for variables in different domains, we will design different evolution approaches. For the optimization problems (20) and (21), the number of dimension for the relay selection scheme is $M$ ( $M$ represents the number of IDT-IDR transmission pairs). The number of continuous variables, i.e., power splitting ratio, dynamic $\mathrm{EH}$ ratio, and power sharing ratio, is three. Hence, the number of optimization problem dimension is $(M+3)$. In an $(M+3)$-dimensional space $((M+3)$ represents the number of dimension for the optimization problem), there are $H$ quantum whales which are used to find the optimal solution. For each quantum whale, it consists of $(M+3)$ quantum bits. The first $M$ dimensions are used to optimize discrete variables, and the final three dimensions are used for optimizing continuous variables. Both discrete variables and continuous variables will evolve in different ways. The $h$ th $(h=1,2, \ldots, H)$ quantum whale $\mathbf{x}_{h}^{t}$ in the $t$ th iteration is given by

$$
\mathbf{x}_{h}^{t}=\left[\begin{array}{l}
\varphi_{h 1}^{t}, \varphi_{h 2}^{t}, \ldots, \varphi_{h i}^{t}, \ldots, \varphi_{h M}^{t}, \varphi_{h(M+1)}^{t}, \varphi_{h(M+2)}^{t}, \varphi_{h(M+3)}^{t} \\
\phi_{h 1}^{t}, \phi_{h 2}^{t}, \ldots, \phi_{h i}^{t}, \ldots, \phi_{h M}^{t}, \phi_{h(M+1)}^{t}, \phi_{h(M+2)}^{t}, \phi_{h(M+3)}^{t}
\end{array}\right],
$$

where $i=1,2, \ldots, M+3$ and $\left|\varphi_{h i}^{t}\right|^{2}+\left|\phi_{h i}^{t}\right|^{2}=1$. To lower computational complexity and improve evolutionary efficiency of QWOA, we define $0 \leq \varphi_{h i}^{t} \leq 1$ and $0 \leq \phi_{h i}^{t} \leq 1$. For each $\varphi_{h i}^{t}, \varphi_{h i}^{t}=\sqrt{1-\left(\phi_{h i}^{t}\right)^{2}}$, each $\phi_{h i}^{t}$ can be computed by its corresponding $\varphi_{h i}^{t}$. Hence, $\mathbf{x}_{h}^{t}$ is simply expressed in the following:

$$
\begin{aligned}
\mathbf{x}_{h}^{t} & =\left[\varphi_{h 1}^{t}, \varphi_{h 2}^{t}, \ldots, \varphi_{h i}^{t}, \ldots, \varphi_{h M}^{t}, \varphi_{h(M+1)}^{t}, \varphi_{h(M+2)}^{t}, \varphi_{h(M+3)}^{t}\right] \\
& =\left[x_{h 1}^{t}, x_{h 2}^{t}, \ldots, x_{h i}^{t}, \ldots, x_{h M}^{t}, x_{h(M+1)}^{t}, x_{h(M+2)}^{t}, x_{h(M+3)}^{t}\right]
\end{aligned}
$$

where $i=1,2, \ldots, M+3$ and $0 \leq x_{h i}^{t} \leq 1$. Each $x_{h i}^{t}$ is the $i$ th quantum bit of the $h$ th quantum whale $\mathbf{x}_{h}^{t}$. 
For the $h$ th quantum whale $\mathbf{x}_{h}^{t}, x_{h i}^{t}(i=1,2, \ldots, M+3)$ should be measured to its own definition domain $\bar{x}_{h i}^{t}$ according to the following rules:

$$
\begin{aligned}
& \bar{x}_{h i}^{t}=\left\{\begin{array}{l}
1, \vartheta_{h i}^{t}>x_{h i}^{t}, \\
0, \vartheta_{h i}^{t} \leq x_{h i}^{t}, \quad i=1,2, \ldots, M,
\end{array}\right. \\
& \bar{x}_{h i}^{t}=l_{i}+x_{h i}^{t}\left(u_{i}-l_{i}\right), \quad i=M+1, M+2, M+3,
\end{aligned}
$$

where $\vartheta_{h i}^{t}$ is a uniform random number which is distributed from zero to one, $l_{i}$ is the $i$ th dimensional variant's lower bound for continuous variables, and $u_{i}$ is the $i$ th dimensional variant's upper bound for the continuous variables. After the process of mapping, we calculate fitness of each quantum whale according to the fitness function. Until the $t$ th iteration, the global optimal quantum whale $\mathbf{p}_{g}^{t}$ of the whole population is shown as $\mathbf{p}_{g}^{t}=\left[p_{g 1}^{t}, p_{g 2}^{t}, \ldots, p_{g i}^{t}, \ldots\right.$, $\left.p_{g M}^{t}, p_{g(M+1)}^{t}, p_{g(M+2)}^{t}, p_{g(M+3)}^{t}\right]$, and its mapping state is $\overline{\mathbf{p}}_{g}^{t}=\left[\bar{p}_{g 1}^{t}, \bar{p}_{g 2}^{t}, \ldots, \bar{p}_{g i}^{t}, \ldots, \bar{p}_{g M}^{t}, \bar{p}_{g(M+1)}^{t}, \bar{p}_{g(M+2)}^{t}, \bar{p}_{g(M+3)}^{t}\right]$.
For the whole population, each quantum whale is evolved in different ways, which contains encircling prey, searching for prey, and bubble-net attacking. For each evolution mode, we will update their quantum rotation angle and quantum bit with different methods. During each iteration, we first calculate the coefficient vector $\mathbf{A}_{h}^{t}=\left[A_{h 1}^{t}, A_{h 2}^{t}, \ldots, A_{h(M+3)}^{t}\right]$ of the $h$ th quantum whale, and the calculation rules can be shown by

$$
A_{h i}^{t}=2 \cdot \xi_{1} \cdot \psi-\psi
$$

where $\xi_{1}$ is a uniform random number that is distributed from zero to one. $\psi=1-t / K$ and $K$ is the maximum iteration number. For the $h$ th quantum whale, we generate a uniform random number $\xi_{2}$ that is distributed from zero to one. When $\xi_{2}<0.5$ and $\left|\mathbf{A}_{h}^{t}\right|<1(|\bullet|$ is the modulus function), the $h$ th quantum whale will encircle the prey and the $i$ th $(i=1,2, \ldots, M+3)$ quantum rotation angle and the $i$ th $(i=1,2, \ldots, M+3)$ quantum bit of the $h$ th quantum whale are updated as

$$
\begin{aligned}
& \theta_{h i}^{t+1}= \begin{cases}\left|\mathbf{A}_{h}^{t}\right| \cdot \xi_{3} \cdot\left(\bar{p}_{g i}^{t}-\bar{x}_{h i}^{t}\right), & \text { if } i=1,2, \ldots, M, \\
\left|\mathbf{A}_{h}^{t}\right| \cdot \xi_{3} \cdot\left(p_{g i}^{t}-x_{h i}^{t}\right), & \text { if } i=M+1, M+2, M+3,\end{cases} \\
& \mu_{h i}^{t+1}= \begin{cases}\operatorname{abs}\left(x_{h i}^{t} \cdot \cos \theta_{h i}^{t+1}-\sqrt{1-\left(x_{h i}^{t}\right)^{2}} \cdot \sin \theta_{h i}^{t+1}\right), & \text { if } i=1,2, \ldots, M, \\
\operatorname{abs}\left(x_{h i}^{t} \cdot \cos \theta_{h i}^{t+1}+\sqrt{1-\left(x_{h i}^{t}\right)^{2}} \cdot \sin \theta_{h i}^{t+1}\right), & \text { if } i=M+1, M+2, M+3,\end{cases}
\end{aligned}
$$

where $\xi_{3}$ is a uniform random number that is distributed from zero to one. $\operatorname{abs}(\cdot)$ is the absolute value function. When $\xi_{2}<0.5$ and $\left|\mathbf{A}_{h}^{t}\right| \geq 1$, the $h$ th quantum whale will enter searching for the prey mode. In this mode, the quantum rotation angle is given by

$$
\theta_{h i}^{t+1}= \begin{cases}\left|\mathbf{A}_{h}^{t}\right| \cdot \xi_{4} \cdot\left(\bar{x}_{a i}^{t}-\bar{x}_{h i}^{t}\right), & \text { if } i=1,2, \ldots, M, \\ \left|\mathbf{A}_{h}^{t}\right| \cdot \xi_{4} \cdot\left(x_{a i}^{t}-x_{h i}^{t}\right), & \text { if } i=M+1, M+2, M+3,\end{cases}
$$

where $\xi_{4}$ is a uniform random number, $a \in\{1,2, \ldots, H\}$ is a random integer, and the quantum bit of each quantum whale is updated as (28).

If $\xi_{2}$ is no less than 0.5 , the quantum whale will take bubble-net attacking behaviour. In this situation, the quantum rotation angle and the quantum bit can be shown as

$$
\begin{aligned}
& \theta_{h i}^{t+1}= \begin{cases}c_{2} \cdot \xi_{5} \cdot\left(\bar{p}_{g i}^{t}-\bar{x}_{h i}^{t}\right) \cdot e^{b \xi_{6}}, & \text { if } i=1,2, \ldots, M, \\
c_{1} \cdot \xi_{5} \cdot\left(p_{g i}^{t}-x_{h i}^{t}\right) \cdot e^{b \xi_{6}}, \quad \text { if } i=M+1, M+2, M+3,\end{cases} \\
& \mu_{h i}^{t+1}= \begin{cases}\operatorname{abs}\left(x_{h i}^{t} \cdot \cos \theta_{h i}^{t+1}-\sqrt{1-\left(x_{h i}^{t}\right)^{2}} \cdot \sin \theta_{h i}^{t+1}\right), & \text { if } i=1,2, \ldots, M, \\
\operatorname{abs}\left(x_{h i}^{t} \cdot \cos \theta_{h i}^{t+1}+\sqrt{1-\left(x_{h i}^{t}\right)^{2}} \cdot \sin \theta_{h i}^{t+1}\right), & \text { if } i=M+1, M+2, M+3,\end{cases}
\end{aligned}
$$

where $\xi_{5}$ is a uniform random number that is distributed from zero to one, $c_{1}, c_{2}$, and $b$ are constants which determine the radius of bubble-net, and $\xi_{6}$ is a uniform random number which is distributed from -1 to 1 .
The quantum whale $\boldsymbol{\mu}_{h}^{t+1}(h=1,2, \ldots, H)$ is measured to its definition domain $\overline{\boldsymbol{\mu}}_{h}^{t+1}$ according to (24) and (25). Then, the fitness of $\overline{\boldsymbol{\mu}}_{h}^{t+1}$ is computed by fitness function. If the fitness of $\overline{\boldsymbol{\mu}}_{h}^{t+1}$ is better than that of $\overline{\mathbf{x}}_{h}^{t}, \mathbf{x}_{h}^{t+1}=\boldsymbol{\mu}_{h}^{t+1}$; else, 
$\mathbf{x}_{h}^{t+1}=\mathbf{x}_{h}^{t}$. At last, the best quantum whale of current iteration is used to update the global optimal quantum whale.

4.2. Throughput Optimization Process Based on QWOA. QWOA can optimize problem of maximizing IDT-IDR transmission pairs' average throughput or network sum throughput in CEH-IoT-SS. We convert the maximizing throughput problem into the process of finding global optimal quantum whale. The fitness function is set as $f\left(\overline{\mathbf{x}}_{h}^{t}\right)= \begin{cases}R\left(\overline{\mathbf{x}}_{h}^{t}\right), & \text { satisfy constraint condition } \\ 0, & \text { else }\end{cases}$ throughput optimization process based on QWOA is presented in Algorithm 1:

\section{Simulation Results and Analysis}

In this part, the performance of proposed QWOA for different communication scenarios is investigated. The simulation results are divided into two parts. In the first part, we compare the optimization results of proposed QWOA and other intelligent algorithms for different communication needs. In fact, since existing algorithms cannot optimize both the continuous problems and discrete problems, we will give the comparison results of QWOA and the circumstances where the classical continuous algorithms are applied to the proposed problems directly. For the second simulation part, we aim to study the impact of different system parameters on the whole system according to different objective functions. In the simulation, PT and PR are located at $(0,1)$ and $(2,1)$ on $X-Y$ plane. For the IDT-IDR transmission pairs, all IDTs are generated in a circle and the center of the circle is $(1,1)$ and the radius of the circle is 0.5 , while IDRs are located in a circle in which center and radius are $(1,0)$ and 0.5 , respectively. The factor of path loss $\ell$ is equal to $3[40]$ and $\rho=1$. For all nodes, the power of AWGN is the same, i.e., $w_{1}=w_{2}=w_{3}=w_{4}=w_{5}=10^{-1}$. In order to not lose generality, the $\mathrm{EH}$ rate of each IDT is the same. Unless otherwise stated, $\quad Y_{\mathrm{PT}}=10, \quad Y_{\mathrm{IDT}}=Y_{\mathrm{IDT}_{m}}=10 \quad(m=1,2, \ldots, M)$, $R_{\mathrm{PR}}^{\text {require }}=0.3$, and the number of IDT-IDR pairs is 10 . All simulation results are average of 500 trials.

5.1. Comparison Simulation Result of QWOA and Other Intelligent Algorithms. In this section, we show the performance of QWOA and other intelligent algorithms for the proposed two problems, which are sum throughput and IDT-IDR transmission pairs' average throughput. Figures 3-6 show the simulation results when the objective function is maximizing the average throughput of IDT-IDR transmission pairs, i.e., equation (20). Meanwhile, Figures 7-9 investigate comparison results for maximizing sum throughput, and the objective function is equation (21). In order to intuitively compare the performance of the traditional algorithm and the proposed algorithm, we apply some continuous intelligent algorithms to CEH-IoT-SS. The process for optimizing power splitting ratio, dynamic EH ratio, and power sharing ratio by continuous intelligent algorithms is the same as the course of optimizing continuous variables. However, for optimizing the relay selection scheme, we are going to round continuous variables up to integer for the compared continuous intelligent algorithms. Compared algorithms can be shown as follows: whale optimization algorithm (WOA), differential evolutionary algorithm (DEA), and the proposed QWOA. In all simulation results, the maximal iteration number is 500 and the number of population size $H$ is set as 20 . For QWOA, $c_{1}=2, c_{2}=2$, and $b=1.5$. For WOA, the parameter settings can refer to [42]. For DEA, the parameter settings of DEA can refer to [46].

Figures 3 and 7 give the comparison results of QWOA and two classical continuous intelligent algorithms when considering equations (20) and (21) as optimization object, respectively. It is easy to see that QWOA has a faster convergence speed and higher convergence accuracy than WOA and DEA under the same simulation conditions. Since the proposed QWOA uses the thinking of quantum evolution theory and whale hunting characteristics, QWOA is more suitable to solve hybrid optimization problems. For continuous variables and discrete variables, we design different evolution strategies to obtain the solution. The resolving method of traditional WOA is updating individual in the population by some certain equations, and it is easy to get into a local optimum in the early searching stage. From the simulation results, we can see that WOA gets a better solution in the last dozens of iterations. This is because the traditional WOA will process an accurate search due to the searching step length which is generated in a fixed way. However, it is still a local optimum. Unlike WOA, since designed evolution strategies can make full use of the advantages of quantum theory and WOA, the best solution is obtained by QWOA. For DEA, it has disadvantages such as local convergence and randomness and it cannot have a good performance on the proposed problem. To summarize, QWOA can overcome the shortcomings of traditional algorithms and show its advantage in convergence speed and convergence accuracy.

In Figure 4, we investigate the impact of $Y_{\text {IDT }}$ on the IDT-IDR transmission pairs' average throughput. $Y_{\mathrm{PT}}=10$ and $Y_{\text {IDT }}$ varies from 2 to 16 in simulation. It is easy to see that as $Y_{\text {IDT }}$ increases, IDT-IDR transmission pairs' average throughput becomes larger and larger for QWOA. This is because as $Y_{\text {IDT }}$ increases, each IDT can get more energy to store for information transfer. A larger $Y_{\text {IDT }}$ can make each IDT use less time to store enough energy. However, due to the fact that WOA and DEA cannot find the best solution, IDT-IDR transmission pairs' average throughput for WOA and DEA is not increasing all the time as $Y_{\text {IDT }}$ becomes larger. Simulation results show that QWOA can find the best solution for the whole network and have a better performance compared with WOA and DEA for different $Y_{\text {IDT }}$.

Figure 5 considers the circumstance where $Y_{\mathrm{IDT}}=10$ and $Y_{\mathrm{PT}}$ increases from 2 to 16 . For different simulation situations, QWOA has the best performance all the time. The average throughput of IDT-IDR transmission pairs becomes larger and larger when $Y_{\mathrm{PT}}$ obtains a higher value. It is easy to understand. As $Y_{\mathrm{PT}}$ increases, $\mathrm{PT}$ can collect more energy for the same time and PT has more energy for transmitting its own information. In addition, each IDT can store more energy from PT as $Y_{\mathrm{PT}}$ increases. More energy of IDTs can bring a bigger SINR for IDT-IDR transmission pairs and more easily satisfy the need of the primary network. 
(1) Input system parameters of CEH-IoT-SS, the fitness function;

(2) Initialize the initial population of $H$ quantum whales;

(3) $t=1 / /$ the first iteration;

(4) Measure each quantum whale to the definition domain according to (24) and (25);

(5) Compute fitness of each quantum whale in accordance with the fitness function and find out the global optimal quantum whale $\mathbf{p}_{g}^{t}$

(6) while $t \leq K$

(7) Calculate the coefficient vector of each quantum whale

(8) if $\xi_{2}<0.5$ and $\left|\mathbf{A}_{h}^{t}\right|<1$

(9) Update the quantum whale through (27) and (28)

(10) else if $\xi_{2}<0.5$ and $\left|\mathbf{A}_{h}^{t}\right| \geq 1$

(11) Update the quantum whale through (29) and (28)

(12) else

(13) Update the quantum whale through (30) and (31)

(14) end if

(15) end if

(16) Measure each quantum whale and compute the fitness according to the fitness function;

(17) Update the quantum whale and the global optimal quantum whale according to the fitness;

(18) Set $t=t+1$;

(19) end while

(20) According to the global optimal quantum whale, obtain the power splitting ratio, dynamic EH ratio, power sharing ratio, and relay selection scheme after $K$ iterations;

(21) Output: Power splitting ratio, dynamic EH ratio, power sharing ratio, and relay selection scheme.

Algorithm 1: Throughput optimization process based on QWOA.

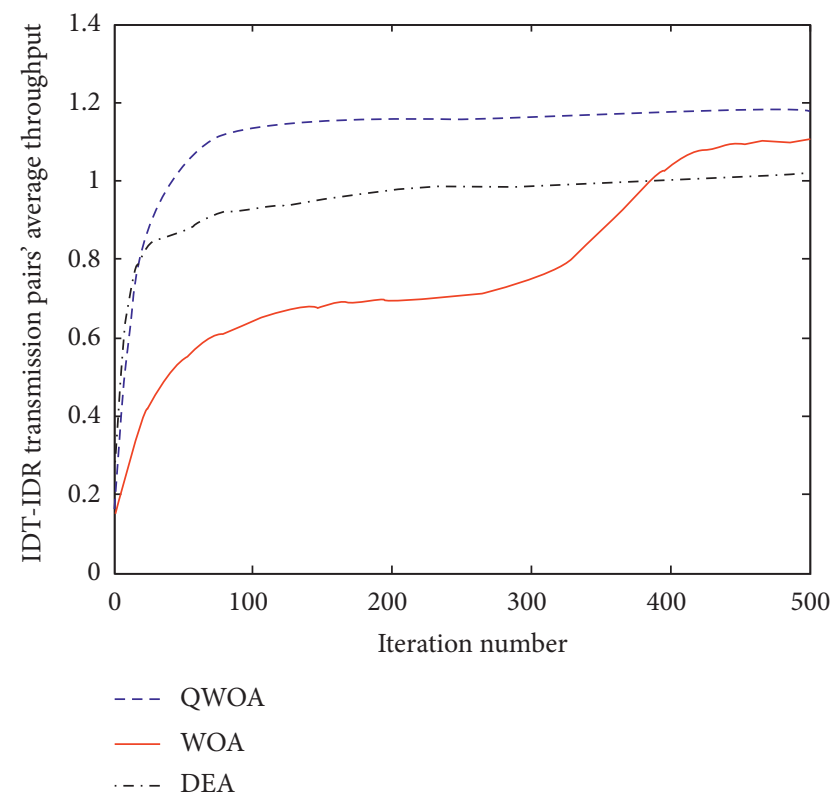

FIGURE 3: Comparison of convergence performance for QWOA, WOA, and DEA (IDT-IDR transmission pairs' average throughput).

Figure 6 shows the IDT-IDR transmission pairs' average throughput with different $R_{\mathrm{PR}}^{\text {require }} \cdot R_{\mathrm{PR}}^{\text {require }}$ varies from 0.1 to 0.8. Simulation results give the performance of QWOA, WOA, and DEA when $R_{\mathrm{PR}}^{\text {require }}$ increases. For any $R_{\mathrm{PR}}^{\text {require }}$, the proposed QWOA can show the best performance all the time. From the simulation results, we can draw a conclusion that the average throughput of IDT-IDR transmission pairs

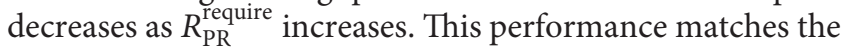
theory. The power need of the primary network is increasing as $R_{\mathrm{PR}}^{\text {require }}$ reaches a larger value. Since the power for

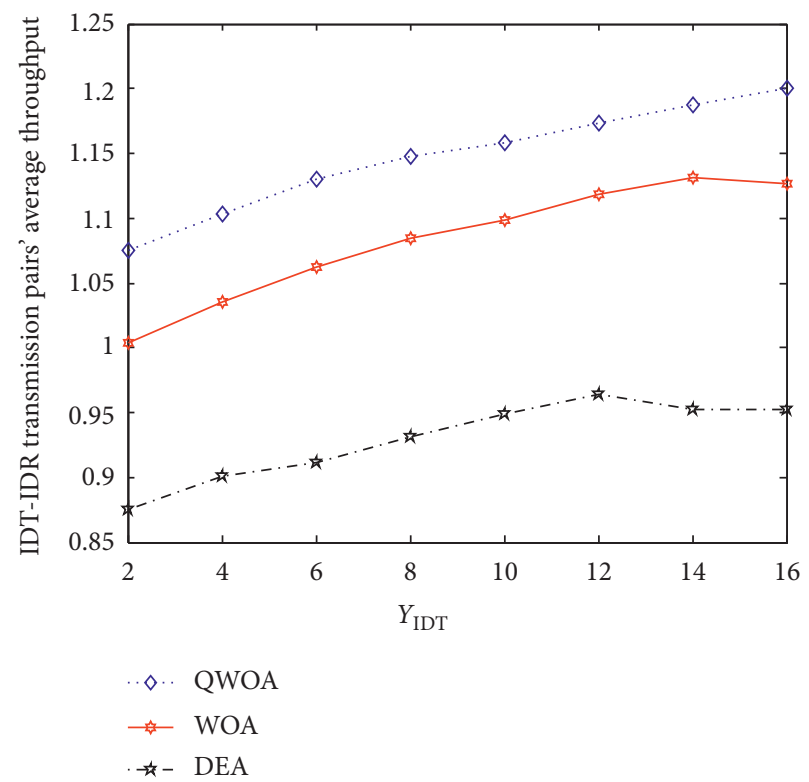

FIGURE 4: IDT-IDR transmission pairs' average throughput of three schemes with different $Y_{\text {IDT }}$.

transmission of the primary network becomes larger, the power that IDT can use becomes less. Therefore, the average throughput of IDT-IDR transmission pairs becomes less.

Figure 8 considers the sum throughput of the whole system as $Y_{\mathrm{PT}}$ increases. $Y_{\mathrm{PT}}$ increases from 2 to 16 . It is clear that the sum throughput reaches a higher value when $Y_{\mathrm{PT}}$ increases. When $Y_{\mathrm{PT}}$ becomes larger, the energy collected by PT increases in one time slot. More energy of PT not only helps information transfer of the primary network but also provides more power for IDT-IDR transmission pairs and 


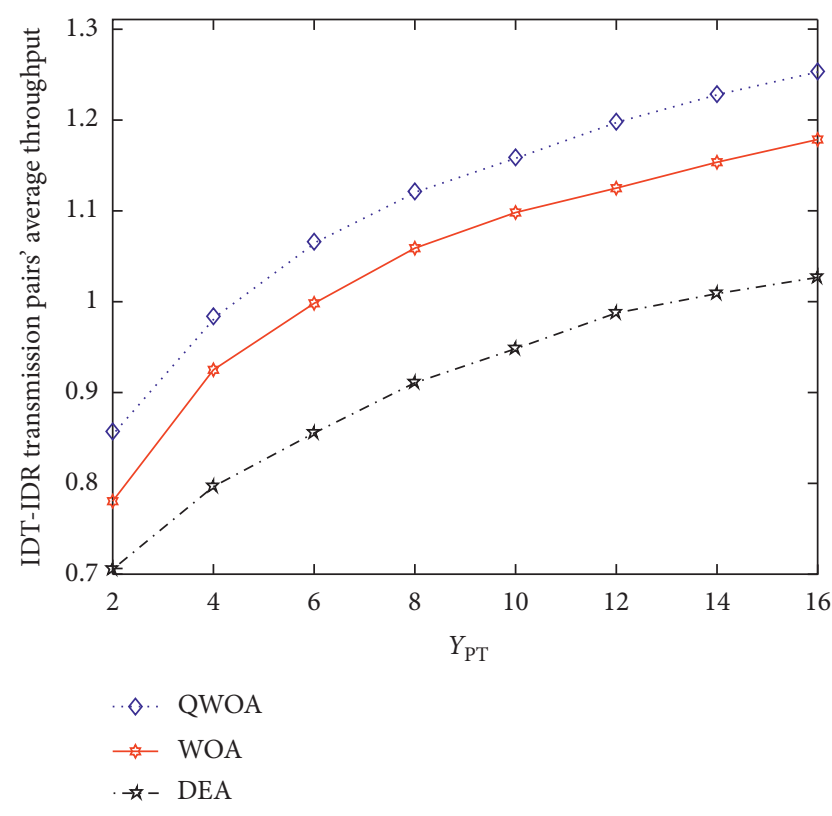

FIGURE 5: IDT-IDR transmission pairs' average throughput of three schemes with different $Y_{\mathrm{PT}}$.

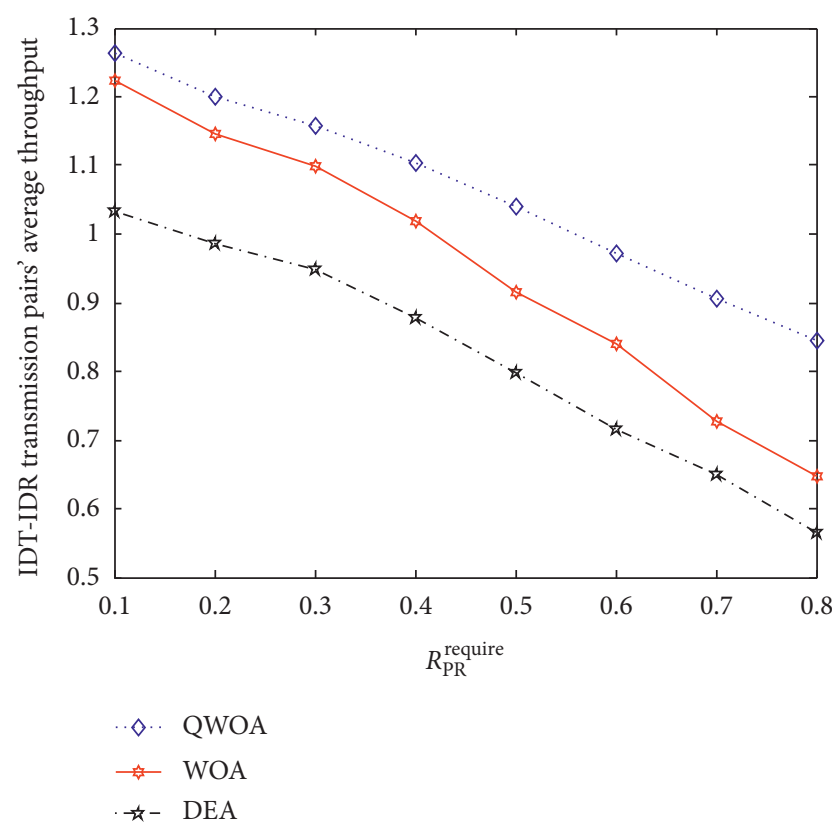

FIGURE 6: IDT-IDR transmission pairs' average throughput of three schemes with different $R_{\mathrm{PR}}^{\text {require }}$.

each IDTs can share more energy to process cooperative communication and its own information transmission. Besides, by comparing with WOA and DEA, we can find that QWOA can get the best simulation results all the time.

In Figure 9, the impact of $R_{\mathrm{PR}}^{\text {require }}$ on the whole system is presented. For a certain $R_{\mathrm{PR}}^{\text {require }}$, QWOA can obtain the best performance compared with WOA and DEA. As $R_{\mathrm{PR}}^{\text {require }}$ increase, the sum throughput of the whole system becomes smaller and smaller. This is because a higher $R_{\mathrm{PR}}^{\text {require }}$ will bring more constraint for the primary network and IDT-IDR transmission

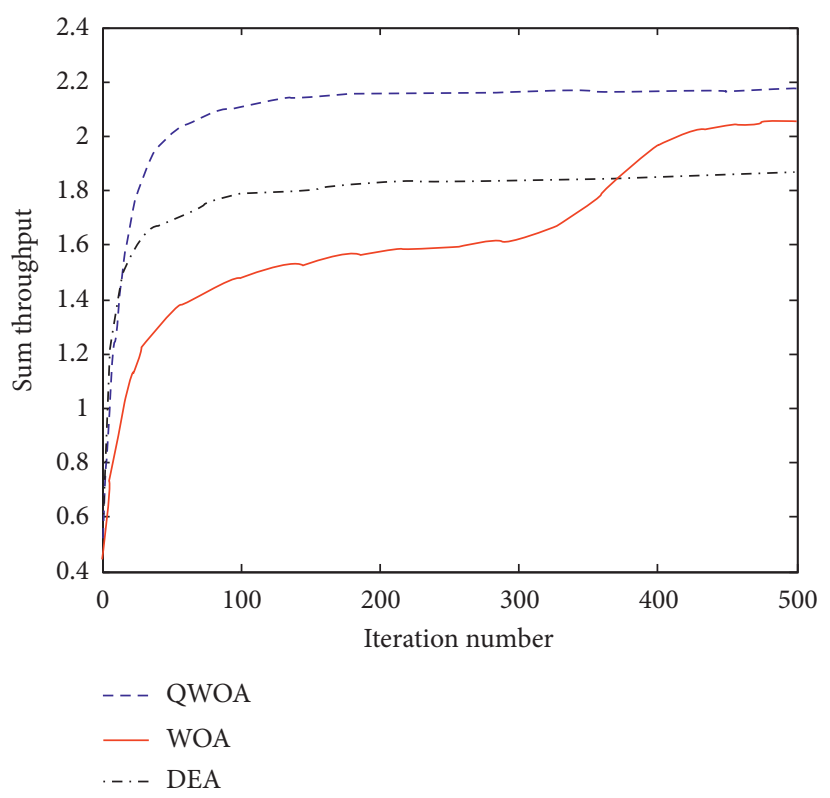

FIGURE 7: Comparison of convergence performance for QWOA, WOA, and DEA (sum throughput).

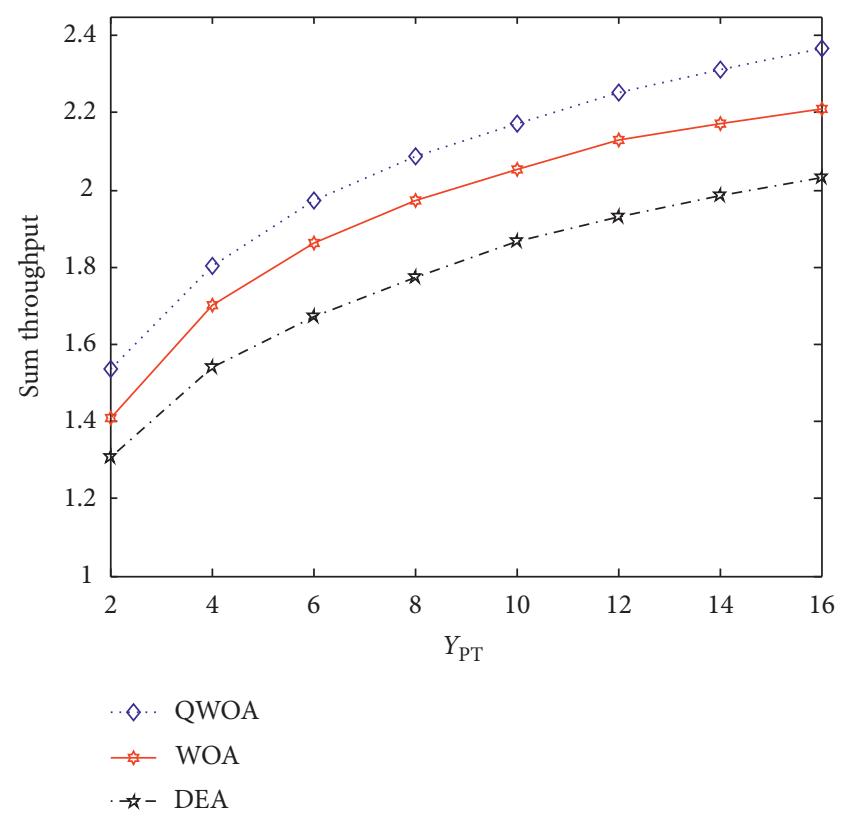

Figure 8: Sum throughput of three schemes with different $Y_{\mathrm{PT}}$.

pairs. The number of solutions for the best power splitting ratio, dynamic $\mathrm{EH}$ ratio, power sharing ratio, and the relay selection decreases with a higher $R_{\mathrm{PR}}^{\text {require }}$. Therefore, the sum throughput becomes smaller with a higher $R_{\mathrm{PR}}^{\text {require }}$. From Figures 3 to 9 , the proposed QWOA can get the best performance all the time. All the simulation results show the advantages of QWOA in convergence speed and convergence accuracy.

5.2. Simulations of Different Parameters Based on $Q W O A$ for CEH-IoT-SS. In this part, the impact of partial different parameters on two proposed objective functions for 


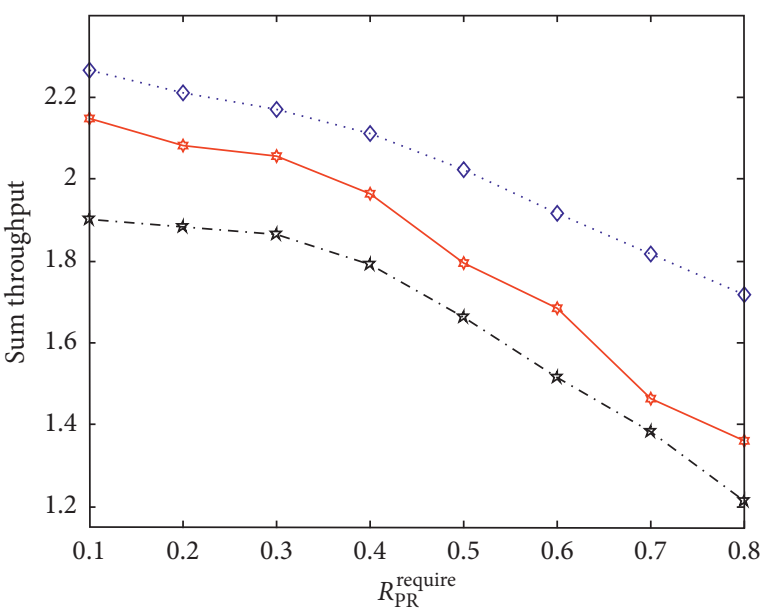

$$
\begin{aligned}
& . \diamond \cdot \text { QWOA } \\
& - \text { WOA } \\
& \rightarrow-\text { DEA }
\end{aligned}
$$

FIGURE 9: Sum throughput of three schemes with different $R_{\mathrm{PR}}^{\text {require }}$.

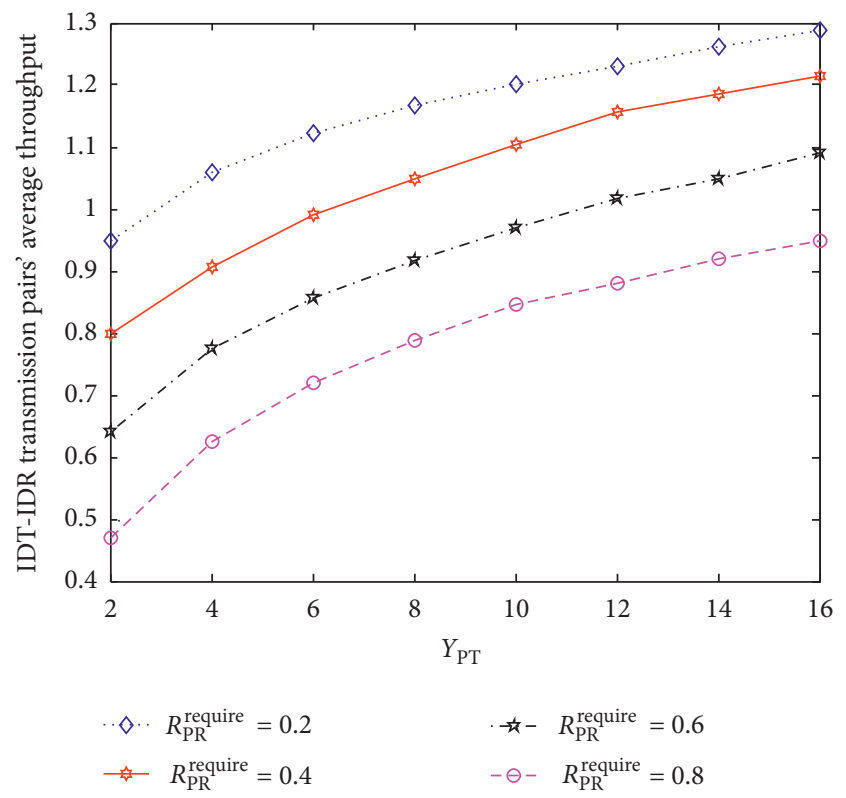

Figure 10: IDT-IDR transmission pairs' average throughput of different $Y_{\mathrm{PT}}$ and $R_{\mathrm{PR}}^{\text {require }}$.

CEH-IoT-SS is presented. We show part of simulation results because of the similarity of parameter effects.

Figure 10 presents the IDT-IDR transmission pairs' average throughput when $R_{\mathrm{PR}}^{\text {require }}=0.2, R_{\mathrm{PR}}^{\text {require }}=0.4, R_{\mathrm{PR}}^{\text {require }}=0.6$, and $R_{\mathrm{PR}}^{\text {require }}=0.8$. The $\mathrm{EH}$ rate, i.e., $Y_{\mathrm{PT}}$ varies from 2 to 16 . Due to the fact that a higher $Y_{\mathrm{PT}}$ can help PT store more energy at the same time and IDT can share more energy from PT, the average throughput of IDT-IDR transmission pairs becomes higher and higher when $Y_{\mathrm{PT}}$ increases. Meanwhile, the more need of the primary network will bring part of energy from IDT-IDR transmission pairs. Hence, IDT-IDR transmission pairs' average throughput will decrease with a higher $R_{\mathrm{PR}}^{\text {require }}$.

Figure 11 considers the impact of $Y_{\mathrm{IDT}}$ and $Y_{\mathrm{PT}}$ on IDTIDR transmission pairs' average throughput. $Y_{\mathrm{PT}}$ varies from

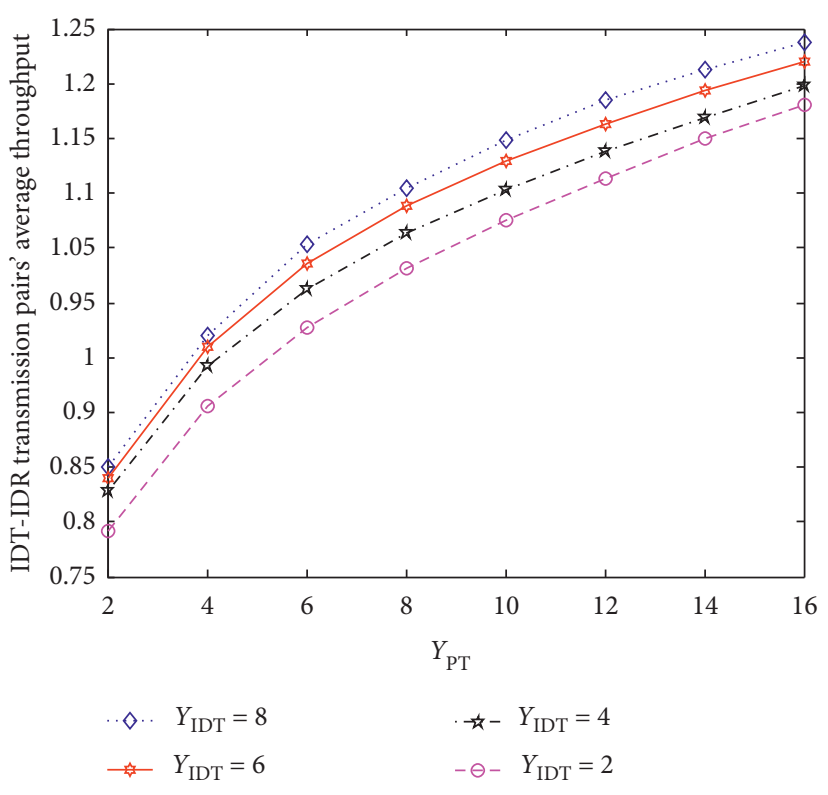

FIGURE 11: IDT-IDR transmission pairs' average throughput of different $Y_{\mathrm{PT}}$ and $Y_{\mathrm{IDT}}$.

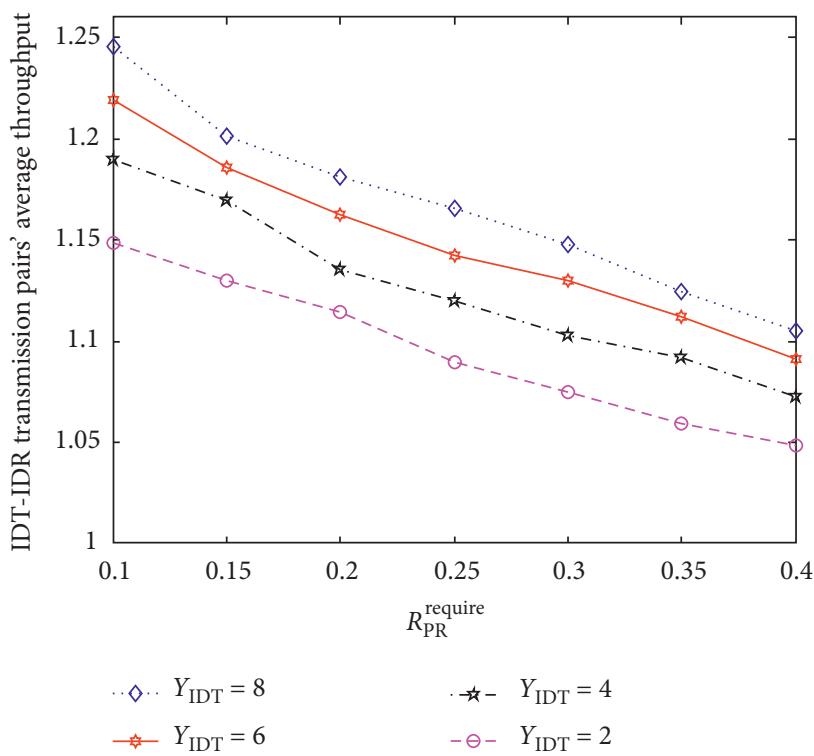

FIGURE 12: IDT-IDR transmission pairs' average throughput of different $R_{\mathrm{PR}}^{\text {require }}$ and $Y_{\mathrm{IDT}}$.

2 to $16 . Y_{\mathrm{IDT}}$ is $2,4,6$, and 8 , respectively. From the simulation results, we can easily find that IDT-IDR transmission pairs' average throughput increases larger with a higher $Y_{\text {IDT }}$ and $Y_{\mathrm{PT}}$. For a certain period of time, the higher $Y_{\mathrm{IDT}}$ and $Y_{\mathrm{PT}}$, the more energy will be stored. More energy will bring a higher power to the primary network and IDT-IDR transmission pairs for information transmission and it can increase the throughput for the certain communication problem. Therefore, boosting $Y_{\mathrm{IDT}}$ and $Y_{\mathrm{PT}}$ can improve the performance of IDT-IDR transmission pairs.

In Figure 12, the impact of different $R_{\mathrm{PR}}^{\text {require }}$ and $Y_{\mathrm{IDT}}$ on the IDT-IDR transmission pairs is investigated. $R_{\mathrm{PR}}^{\text {require }}$ is increasing from 0.1 to 0.4 , while $Y_{\mathrm{IDT}}$ is $2,4,6$, and 8 , 


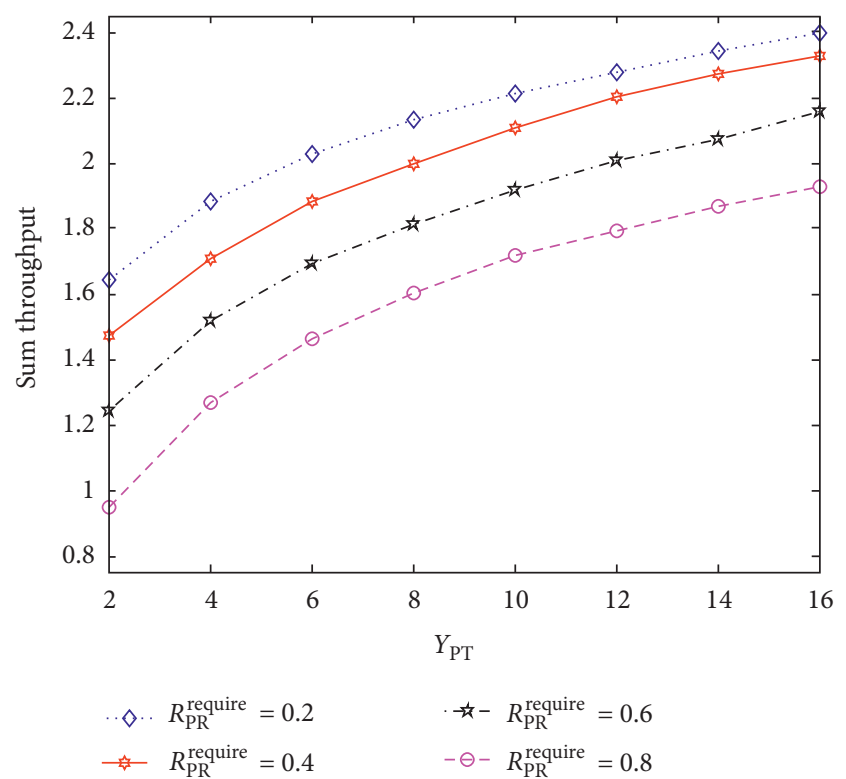

FIGURE 13: Sum throughput of different $Y_{\mathrm{PT}}$ and $R_{\mathrm{PR}}^{\text {require }}$.

respectively. For a certain $Y_{\mathrm{IDT}}$, the average throughput of IDT-IDR transmission pairs decreases with $R_{\mathrm{PR}}^{\text {require }}$ becomes higher and higher. Meanwhile, the average throughput of IDT-IDR transmission pairs increases with a higher $Y_{\text {IDT }}$.

Figure 13 presents the simulation results which consider different $Y_{\mathrm{PT}}$ and $R_{\mathrm{PR}}^{\text {require }}$ for the sum throughput of the primary network and IDT-IDR transmission pairs. In the simulation, $R_{\mathrm{PR}}^{\text {require }}$ is set as $0.2,0.4,0.6$, and 0.8 , respectively. $Y_{\mathrm{PT}}$ varies from 2 to 16 . It is easy to see that a higher $Y_{\mathrm{PT}}$ is conducive to the whole network and a higher $R_{\mathrm{PR}}^{\text {require }}$ will reduce the throughput of the network. To improve $Y_{\mathrm{PT}}$ is a meaningful thing for the whole network. It is obvious that the proposed QWOA can satisfy different throughput requirements of the primary network.

\section{Conclusion}

In this paper, we design a novel $\mathrm{EH}$ and information transmission mode for CEH-IoT-SS. In the network, IDTs can work as relays for PU's information transmission and finish its own information transfer in one time slot. The expressions for the throughput of the primary network and IDT-IDR transmission pairs are mathematically derived. For different communication scenarios, we formulate two objective functions with different communication demands. Since the formulated objective functions are hybrid optimization problems, traditional intelligent algorithms cannot solve it well. A new algorithm-QWOA is proposed to solve it. Compared with the traditional algorithms, QWOA can get the best solution for hybrid optimization problems and various system parameters. Simulation results validate that QWOA has a wide application for different communication scenarios and hybrid problems. In our future work, we will apply our work to other specific IoT scenarios, such as A-IoT, fog computing, and blockchain.

\section{Appendix}

For the $m$ th IDR, thereceived signal in the third phase is shown by

$$
\begin{aligned}
& z_{\mathrm{IDR}_{m}}=\sqrt{P_{\mathrm{IDT}_{m}}} G_{\mathrm{IDT}_{m}, \mathrm{IDR}_{m}} z_{\mathrm{IDT}_{m}}+\sum_{i=1, i \neq m}^{M} \sqrt{P_{\mathrm{IDT}_{i}}} G_{\mathrm{IDT}_{i}, \mathrm{IDR}_{m}} z_{\mathrm{IDT}_{i}}+n_{5} \\
& =\sqrt{P_{\mathrm{IDT}_{m}}} G_{\mathrm{IDT}_{m}, \mathrm{IDR}_{m}} z_{\mathrm{IDT}_{m}}+\sum_{i=1, i \neq m}^{M} b_{i} G_{\mathrm{IDT}_{i}, \mathrm{IDR}_{m}} \sqrt{P_{\mathrm{IDT}_{i}}(1-\sigma)} z_{\mathrm{IDT}_{i}} \\
& +\sum_{i=1, i \neq m}^{M} b_{i} \frac{\sqrt{\left(2 Y_{\mathrm{PT}} \alpha /(1-\alpha)\right) \sigma P_{\mathrm{IDT}_{i}}(1-\beta)} G_{\mathrm{IDT}_{i}, \mathrm{IDR}_{m} G_{\mathrm{PT} \mathrm{IDT}_{i}}}}{\sqrt{\left(2 Y_{\mathrm{PT}} \alpha /(1-\alpha)\right)(1-\beta)\left|G_{\mathrm{PT}, \mathrm{IDT}_{i}}\right|^{2}+(1-\beta) w_{1}+w_{3}}} x \\
& +\sum_{i=1, i \neq m}^{M} b_{i} \frac{\sqrt{\sigma P_{\mathrm{IDT}_{i}}(1-\beta)} G_{\mathrm{IDT}_{i}, \mathrm{IDR}_{m}}}{\sqrt{\left(2 Y_{\mathrm{PT}} \alpha /(1-\alpha)\right)(1-\beta)\left|G_{\mathrm{PT}_{\mathrm{IDT}}}\right|^{2}+(1-\beta) w_{1}+w_{3}}} n_{1} \\
& +\sum_{i=1, i \neq m}^{M} b_{i} \frac{\sqrt{\sigma P_{\mathrm{IDT}_{i}}} G_{\mathrm{IDT}_{i}, \mathrm{IDR}_{m}}}{\sqrt{\left(2 Y_{\mathrm{PT}} \alpha /(1-\alpha)\right)(1-\beta)\left|G_{\left.\mathrm{PT}_{\mathrm{IDT}}\right|_{i}}\right|^{2}+(1-\beta) w_{1}+w_{3}}} n_{3} \\
& +\sum_{i=1, i \neq m}^{M}\left(1-b_{i}\right) G_{\mathrm{IDT}_{i}, \mathrm{IDR}_{m}} \sqrt{P_{\mathrm{IDT}_{i}}} z_{\mathrm{IDT}_{i}}+n_{5}
\end{aligned}
$$

where $n_{5}$ is the AWGN with power $w_{5} \cdot \sqrt{P_{\mathrm{IDT}_{m}}} G_{\mathrm{IDT}_{m}, \mathrm{IDR}_{m}} z_{\mathrm{IDT}_{m}}$ is a useful signal part for the $m$ th IDR and the other parts are interference signals. According to (5), the $m$ th IDR has received information $x$ of $\mathrm{PT}$ in the 
second phase. Therefore, the signal interference from PT, i.e., $\sum_{i=1, i \neq m}^{M} b_{i}\left[\sqrt{\left(2 Y_{\mathrm{PT}} \alpha /(1-\alpha)\right) \sigma P_{\mathrm{IDT}_{i}}(1-\beta)} G_{\mathrm{IDT}_{i}}, \operatorname{IDR}_{m}\right.$ $\left.G_{\mathrm{PT}, \mathrm{IDT}_{i}} / \sqrt{\left(2 Y_{\mathrm{PT}} \alpha /(1-\alpha)\right)(1-\beta)\left|G_{\mathrm{PT}, \mathrm{IDT}_{i}}\right|^{2}+(1-\beta) w_{1}+w_{3}}\right] x$ can be removed from (A.1) and the expression for the residual part is shown by

$$
\begin{aligned}
& z_{\mathrm{IDR}_{m}}=\sqrt{P_{\mathrm{IDT}_{m}}} G_{\mathrm{IDT}_{m}, \mathrm{IDR}_{m}} z_{\mathrm{IDT}_{m}}+\sum_{i=1, i \neq m}^{M} b_{i} G_{\mathrm{IDT}_{i}, \mathrm{IDR}_{m}} \sqrt{P_{\mathrm{IDT}_{i}}(1-\sigma)} z_{\mathrm{IDT}_{i}} \\
& +\sum_{i=1, i \neq m}^{M} b_{i} \frac{\sqrt{\sigma P_{\mathrm{IDT}_{i}}(1-\beta)} G_{\mathrm{IDT}_{i}, \mathrm{IDR}_{m}}}{\sqrt{\left(2 Y_{\mathrm{PT}} \alpha /(1-\alpha)\right)(1-\beta)\left|G_{\mathrm{PT}_{\mathrm{IDT}}}\right|^{2}+(1-\beta) w_{1}+w_{3}}} n_{1} \\
& +\sum_{i=1, i \neq m}^{M} b_{i} \frac{\sqrt{\sigma P_{\mathrm{IDT}_{i}}} G_{\mathrm{IDT}_{i}, \mathrm{IDR}_{m}}}{\sqrt{\left(2 Y_{\mathrm{PT}} \alpha /(1-\alpha)\right)(1-\beta)\left|G_{\mathrm{PT}_{\mathrm{IDT}}}\right|^{2}+(1-\beta) w_{1}+w_{3}}} n_{3} \\
& +\sum_{i=1, i \neq m}^{M}\left(1-b_{i}\right) G_{\mathrm{IDT}_{i}, \mathrm{IDR}_{m}} \sqrt{P_{\mathrm{IDT}_{i}}} z_{\mathrm{IDT}_{i}}+n_{5}
\end{aligned}
$$

The SINR of the $m$ th IDR is shown as follows:

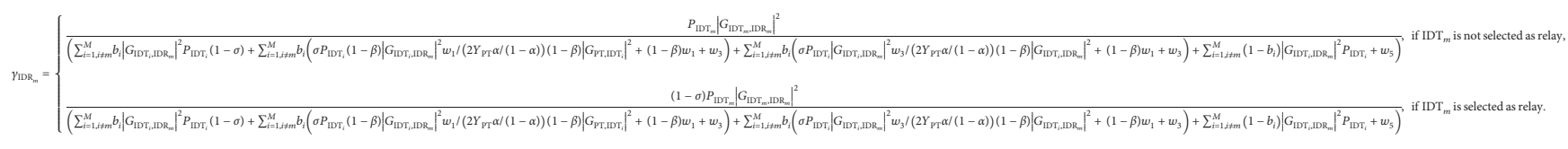

According to (9) and (A.3), the throughput of the $m$ th IDR is given by

$$
\begin{aligned}
& R_{\mathrm{IDR}_{m}}=\frac{1-\alpha}{2} \log _{2}\left(1+\gamma_{\mathrm{IDR}_{m}}\right)=\frac{1-\alpha}{2} \log _{2}\left(1+\frac{\text { Signal }_{\mathrm{IDR}_{m}}}{\text { Interference }_{\mathrm{IDR}_{m}}}\right), \\
& \text { Signal }_{\mathrm{IDR}_{m}}= \begin{cases}\frac{2 \alpha Y_{\mathrm{IDT}_{m}}+2 \rho Y_{\mathrm{PT}} \alpha \beta\left|G_{\mathrm{PT}, \mathrm{IDT}_{m}}\right|^{2}}{1-\alpha}\left|G_{\mathrm{IDT}_{m}, \mathrm{IDR}_{m}}\right|^{2}, & \text { if } \mathrm{IDT}_{m} \text { is not selected as relay, } \\
\frac{2 \alpha Y_{\mathrm{IDT}_{m}}+2 \rho Y_{\mathrm{PT}} \alpha \beta\left|G_{\mathrm{PT}, \mathrm{IDT}_{m}}\right|^{2}}{1-\alpha}(1-\sigma)\left|G_{\mathrm{IDT}_{m}, \mathrm{IDR}_{m}}\right|^{2}, & \text { if } \mathrm{IDT}_{m} \text { is selected as relay, }\end{cases} \\
& \text { Interference }_{\mathrm{IDR}_{m}}=\sum_{i=1, i \neq m}^{M} b_{i}\left|G_{\mathrm{IDT}_{i}, \mathrm{IDR}_{m}}\right|^{2} \frac{2 \alpha Y_{\mathrm{IDT}_{i}}+2 \rho Y_{\mathrm{PT}} \alpha \beta\left|G_{\mathrm{PT}, \mathrm{IDT}_{i}}\right|^{2}}{1-\alpha}(1-\sigma) \\
& +\sum_{i=1, i \neq m}^{M} b_{i} \frac{\sigma\left(\left(2 \alpha Y_{\mathrm{IDT}_{i}}+2 \rho Y_{\mathrm{PT}} \alpha \beta\left|G_{\mathrm{PT}, \mathrm{IDT}_{i}}\right|^{2}\right) /(1-\alpha)\right)(1-\beta)\left|G_{\mathrm{IDT}_{i}, \mathrm{IDR}_{m}}\right|^{2} w_{1}}{\left(2 Y_{\mathrm{PT}} \alpha /(1-\alpha)\right)(1-\beta)\left|G_{\mathrm{PT}, \mathrm{IDT}}\right|^{2}+(1-\beta) w_{1}+w_{3}} \\
& +\sum_{i=1, i \neq m}^{M} b_{i} \frac{\sigma\left(\left(2 \alpha Y_{\mathrm{IDT}_{i}}+2 \rho Y_{\mathrm{PT}} \alpha \beta\left|G_{\mathrm{PT}, \mathrm{IDT}_{i}}\right|^{2}\right) /(1-\alpha)\right)\left|G_{\mathrm{IDT}_{i}, \mathrm{IDR}_{m}}\right|^{2} w_{3}}{\left(2 Y_{\mathrm{PT}} \alpha /(1-\alpha)\right)(1-\beta)\left|G_{\mathrm{IDT}_{i}, \mathrm{IDR}_{m}}\right|^{2}+(1-\beta) w_{1}+w_{3}} \\
& +\sum_{i=1, i \neq m}^{M}\left(1-b_{i}\right)\left|G_{\mathrm{IDT}_{i}, \mathrm{IDR}_{m}}\right|^{2} \frac{2 \alpha Y_{\mathrm{IDT}_{i}}+2 \rho Y_{\mathrm{PT}} \alpha \beta\left|G_{\mathrm{PT}, \mathrm{IDT}_{i}}\right|^{2}}{1-\alpha}+w_{5} .
\end{aligned}
$$


Similarly, the average throughput of $M$ IDT-IDR transmission pairs in CEH-IoT-SS is expressed by

$$
\begin{aligned}
R_{{\mathrm{IDT}-\mathrm{IDR}_{\text {average }}}} & =\frac{1}{M} \sum_{m=1}^{M} R_{\mathrm{IDR}_{m}}=\frac{1}{M} \sum_{m=1}^{M} \frac{1-\alpha}{2} \log _{2}\left(1+\gamma_{\mathrm{IDR}_{m}}\right) \\
& =\frac{1-\alpha}{2 M} \sum_{m=1}^{M} \log _{2}\left(1+\gamma_{\mathrm{IDR}_{m}}\right) .
\end{aligned}
$$

Combining (A.4) with (A.5), we can obtain the expression of IDT-IDR transmission pairs' average throughput as given in (19). This completes the proof.

\section{Data Availability}

The data (figures) used to support the findings of this study are included within the article. Further details can be provided upon request.

\section{Conflicts of Interest}

The authors declare that they have no conflicts of interest.

\section{Acknowledgments}

This work was supported by the National Natural Science Foundation of China (no. 61571149), Special China Postdoctoral Science Foundation (no. 2015T80325), Fundamental Research Funds for the Central Universities (nos. HEUCFP201808 and HEUCF190801), and China Postdoctoral Science Foundation (no. 2013M530148).

\section{References}

[1] N. Javaid, A. Sher, H. Nasir, and N. Guizani, "Intelligence in IoT-based 5G networks: opportunities and challenges," IEEE Communications Magazine, vol. 56, no. 10, pp. 94-100, 2018.

[2] J. Iannacci, "RF-MEMS for high-performance and widely reconfigurable passive components - a review with focus on future telecommunications, Internet of Things (IoT) and 5G applications," Journal of King Saud University-Science, vol. 29, no. 4, pp. 436-443, 2017.

[3] F. Benkhelifa, K. Tourki, and M.-S. Alouini, "Proactive spectrum sharing for SWIPT in MIMO cognitive radio systems using antenna switching technique," IEEE Transactions on Green Communications and Networking, vol. 1, no. 2, pp. 204-222, 2017.

[4] A. Aijaz and A. H. Aghvami, "Cognitive machine-to-machine communications for Internet-of-Things: a protocol stack perspective," IEEE Internet of Things Journal, vol. 2, no. 2, pp. 103-112, 2015.

[5] P. Rawat, K. D. Singh, and J. M. Bonnin, "Cognitive radio for M2M and Internet of Things: a survey," Computer Communications, vol. 94, pp. 1-29, 2016.

[6] G. Ding, Q. Wu, L. Zhang, Y. Lin, T. A. Tsiftsis, and Y.-D. Yao, "An amateur drone surveillance system based on the cognitive Internet of Things," IEEE Communications Magazine, vol. 56, no. 1, pp. 29-35, 2018.
[7] Z. Li, B. Chang, S. Wang, A. Liu, F. Zeng, and G. Luo, "Dynamic compressive wide-band spectrum sensing based on channel energy reconstruction in cognitive Internet of Things," IEEE Transactions on Industrial Informatics, vol. 14, no. 6, pp. 2598-2607, 2018.

[8] K. Zaheer, M. Othman, M. H. Rehmani, and T. Perumal, "A survey of decision-theoretic models for cognitive Internet of Things (CIoT)," IEEE Access, vol. 6, pp. 22489-22512, 2018.

[9] S. Solanki, P. K. Sharma, and P. K. Upadhyay, "Adaptive link utilization in two-way spectrum sharing relay systems under average interference-constraints," IEEE Systems Journal, vol. 12, no. 4, pp. 3461-3472, 2018.

[10] A. A. Khan, M. H. Rehmani, and A. Rachedi, "Cognitiveradio-based Internet of Things: applications, architectures, spectrum related functionalities, and future research directions," IEEE Wireless Communications, vol. 24, no. 3, pp. 17-25, 2017.

[11] J. Guo, S. Durrani, and X. Zhou, "Performance analysis of arbitrarily-shaped underlay cognitive networks: effects of secondary user activity protocols," IEEE Transactions on Communications, vol. 63, no. 2, pp. 376-389, 2015.

[12] S. Aslam, W. Ejaz, and M. Ibnkahla, "Energy and spectral efficient cognitive radio sensor networks for Internet of Things," IEEE Internet of Things Journal, vol. 5, no. 4, pp. 3220-3233, 2018.

[13] T. P. Do, I. Song, and Y. H. Kim, "Simultaneous wireless transfer of power and information in a decode-and-forward two-way relaying network," IEEE Transactions on Wireless Communications, vol. 16, no. 3, pp. 1579-1592, 2017.

[14] J. Yan and Y. Liu, "A dynamic SWIPT approach for cooperative cognitive radio networks," IEEE Transactions on Vehicular Technology, vol. 66, no. 12, pp. 11122-11136, 2017.

[15] A. A. Nasir, X. Zhou, S. Durrani, and R. A. Kennedy, "Wireless-powered relays in cooperative communications: time-switching relaying protocols and throughput analysis," IEEE Transactions on Communications, vol. 63, no. 5, pp. 1607-1622, 2015.

[16] T. Li, P. Fan, and K. B. Letaief, "Outage probability of energy harvesting relay-aided cooperative networks over Rayleigh fading channel," IEEE Transactions on Vehicular Technology, vol. 65, no. 2, pp. 972-978, 2016.

[17] N. Zhao, S. Zhang, F. R. Yu, Y. Chen, A. Nallanathan, and V. C. M. Leung, "Exploiting interference for energy harvesting: a survey, research issues, and challenges," IEEE Access, vol. 5, pp. 10403-10421, 2017.

[18] B. V. Nguyen, H. Jung, D. Har, and K. Kim, "Performance analysis of a cognitive radio network with an energy harvesting secondary transmitter under Nakagami-m fading," IEEE Access, vol. 6, pp. 4135-4144, 2018.

[19] Z. Yang, W. Xu, Y. Pan, C. Pan, and M. Chen, "Energy efficient resource allocation in machine-to-machine communications with multiple access and energy harvesting for IoT," IEEE Internet of Things Journal, vol. 5, no. 1, pp. 229-245, 2018.

[20] D. T. Hoang, D. Niyato, P. Wang, D. I. Kim, and Z. Han, "Ambient backscatter: a new approach to improve network performance for RF-powered cognitive radio networks," IEEE Transactions on Communications, vol. 65, no. 9, pp. 36593674, 2017.

[21] H. Gao, W. Ejaz, and M. Jo, "Cooperative wireless energy harvesting and spectrum sharing in 5G networks," IEEE Access, vol. 4, pp. 3647-3658, 2016.

[22] P. Maji, S. D. Roy, and S. Kundu, "Secrecy outage analysis in a hybrid cognitive relay network with energy harvesting," 
International Journal of Communication Systems, vol. 30, no. 10, Article ID 3228, 2017.

[23] A. O. Ercan, M. O. Sunay, and I. F. Akyildiz, "RF energy harvesting and transfer for spectrum sharing cellular IoT communications in 5G systems," IEEE Transactions on Mobile Computing, vol. 17, no. 7, pp. 1680-1694, 2018.

[24] X. M. Huang, R. Yu, J. W. Kang, Z. Q. Xia, and Y. Zhang, "Software defined networking for energy harvesting Internet of Things," IEEE Internet of Things Journal, vol. 5, no. 3, pp. 1389-1399, 2018.

[25] A. Caruso, S. Chessa, S. Escolar, X. del Toro, and J. C. Lopez, "A dynamic programming algorithm for high-level task scheduling in energy harvesting IoT," IEEE Internet of Things Journal, vol. 5, no. 3, pp. 2234-2248, 2018.

[26] H.-S. Lee and J.-W. Lee, "Resource and task scheduling for SWIPT IoT systems with renewable energy sources," IEEE Internet of Things Journal, vol. 6, no. 2, pp. 2729-2748, 2019.

[27] D. S. Gurjar, H. H. Nguyen, and H. D. Tuan, "Wireless information and power transfer for IoT applications in overlay cognitive radio networks," IEEE Internet of Things Journal, vol. 6, no. 2, pp. 3257-3270, 2019.

[28] Q. Ju, H. Li, and Y. Zhang, "Power management for kinetic energy harvesting IoT," IEEE Sensors Journal, vol. 18, no. 10, pp. 4336-4345, 2018.

[29] S. S. Kalamkar and A. Banerjee, "Secure communication via a wireless energy harvesting untrusted relay," IEEE Transactions on Vehicular Technology, vol. 66, no. 3, pp. 2199-2213, 2017.

[30] A. Mukherjee, T. Acharya, and M. R. A. Khandaker, "Outage analysis for SWIPT-enabled two-way cognitive cooperative communications," IEEE Transactions on Vehicular Technology, vol. 67, no. 9, pp. 9032-9036, 2018.

[31] X. Zhang, Z. Zhang, J. Xing, R. Yu, P. Zhang, and W. Wang, "Exact outage analysis in cognitive two-way relay networks with opportunistic relay selection under primary user's interference," IEEE Transactions on Vehicular Technology, vol. 64, no. 6, pp. 2502-2511, 2015.

[32] B. Lyu, H. Guo, Z. Yang, and G. Gui, "Throughput maximization for hybrid backscatter assisted cognitive wireless powered radio networks," IEEE Internet of Things Journal, vol. 5, no. 3, pp. 2015-2024, 2018.

[33] S. Atapattu, Y. D. Yindi Jing, H. Hai Jiang, and C. Tellambura, "Relay selection and performance analysis in multiple-user networks," IEEE Journal on Selected Areas in Communications, vol. 31, no. 8, pp. 1517-1529, 2013.

[34] K. Singh, A. Gupta, and T. Ratnarajah, "Energy efficient resource allocation for multiuser relay networks," IEEE Transactions on Wireless Communications, vol. 16, no. 2, pp. 1218-1235, 2017.

[35] S. Sharma, Y. Shi, Y. T. Hou, and S. Kompella, "An optimal algorithm for relay node assignment in cooperative ad hoc networks," IEEE/ACM Transactions on Networking, vol. 19, no. 3, pp. 879-892, 2011.

[36] W. Zhong, G. Chen, S. Jin, and K.-K. Wong, "Relay selection and discrete power control for cognitive relay networks via potential game," IEEE Transactions on Signal Processing, vol. 62, no. 20, pp. 5411-5424, 2014.

[37] A. Zappone, S. Atapattu, M. Di Renzo, J. Evans, and M. Debbah, "Energy-efficient relay assignment and power control in multi-user and multi-relay networks," IEEE Wireless Communications Letters, vol. 7, no. 6, pp. 1070-1073, 2018.

[38] S. Yin, E. Zhang, Z. Qu, L. Yin, and S. Li, "Optimal cooperation strategy in cognitive radio systems with energy harvesting," IEEE Transactions on Wireless Communications, vol. 13, no. 9, pp. 4693-4707, 2014.

[39] O. Elnahas, M. Elsabrouty, O. Muta, and H. Furukawa, "Game theoretic approaches for cooperative spectrum sensing in energy-harvesting cognitive radio networks," IEEE Access, vol. 6, pp. 11086-11100, 2018.

[40] Z. Wang, Z. Chen, B. Xia, L. Luo, and J. Zhou, "Cognitive relay networks with energy harvesting and information transfer: design, analysis, and optimization," IEEE Transactions on Wireless Communications, vol. 15, no. 4, pp. 2562-2576, 2016.

[41] H. Gobjuka and Y. J. Breitbart, "Ethernet topology discovery for networks with incomplete information," IEEE/ACM Transactions on Networking, vol. 18, no. 4, pp. 1220-1233, 2010.

[42] S. Mirjalili and A. Lewis, "The whale optimization algorithm," Advances in Engineering Software, vol. 95, pp. 51-67, 2016.

[43] H. Gao, S. Zhang, Y. Su, M. Diao, and M. Jo, "Joint multiple relay selection and time slot allocation algorithm for the EHabled cognitive multi-user relay networks," IEEE Access, vol. 7, pp. 111993-112007, 2019.

[44] L. Yang, B. Y. Yang, and Y. F. Chen, "Full quantum treatment of Rabi oscillation driven by a pulse train and its application in ion-trap quantum computation," IEEE Journal of Quantum Electronics, vol. 49, no. 8, pp. 641-651, 2013.

[45] P. W. Shor, "Algorithms for quantum computation: discrete logarithms and factoring," in Proceedings 35th Annual Symposium on Foundations of Computer Science, pp. 124-134, Santa Fe, NM, USA, November 1994.

[46] N. N. Qin and J. L. Chen, "An area coverage algorithm for wireless sensor networks based on differential evolution," International Journal of Distributed Sensor Networks, vol. 14, no. 8, Article ID 155014771879673, 2018. 